Comparative Population Studies

Selected Articles in German Vol. 41 (2016): 35-66

(Erstveröffentlichung: 22.08.2017)

\title{
Lebenserwartung in Deutschland auf Basis des Zensus 2011: War der Healthy-Migrant-Effekt nur ein Artefakt?*
}

\author{
Felix zur Nieden, Bettina Sommer
}

Zusammenfassung: Die allgemeine Sterbetafel für 2010/12 des Statistischen Bundesamtes liefert für das vereinte Deutschland erstmals Ergebnisse zur Lebenserwartung auf Basis von Zensusdaten. Der vorliegende Artikel widmet sich deshalb der Frage, wie sich die Korrekturen der Bevölkerungsbestände im Rahmen des Zensus 2011 auf die Messung der Lebenserwartung ausgewirkt haben. Hierfür werden sowohl die amtlichen Sterbetafeln auf Basis der alten Bevölkerungsfortschreibung vor dem Zensus als auch auf Basis der Bevölkerungsdaten des Zensus 2011 analysiert. Die Vorgehensweise zur Berechnung der zensusbereinigten allgemeinen Sterbetafel 2010/12 wird zudem auf eine separate Erstellung von Sterbetafeln für die deutsche und die ausländische Bevölkerung übertragen. Befunde zum sogenannten Healthy-Migrant-Effekt können somit unter Ausschluss möglicher Fehler in der Bevölkerungsfortschreibung diskutiert werden. Diese waren zuvor als Hauptursache für eine deutlich höhere Lebenserwartung der ausländischen Bevölkerung im Vergleich zur deutschen Bevölkerung angeführt worden. Für die Bevölkerung insgesamt und für die deutsche Bevölkerung zeigen sich erwartungsgemäß nur sehr geringfügige Korrekturen der Werte für die Lebenserwartung durch eine Berechnung auf Basis des Zensus. Die Lebenserwartung von ausländischen Frauen und Männern wird durch die Nutzung von Zensusergebnissen jedoch deutlich korrigiert. Ein auf Basis der alten Bevölkerungsdaten gemessener Vorteil in der Lebenserwartung bei Geburt von über 5 Jahren, muss zensusbasiert auf etwa 2,9 Jahre bei den Männern und 2,1 Jahre bei den Frauen korrigiert werden. Der Healthy-Migrant-Effekt kann somit nicht ausschließlich auf Datenartefakte der alten Bevölkerungsfortschreibung zurückgeführt werden, da sich auch zensusbereinigt deutliche Überlebensvorteile der ausländischen Bevölkerung zeigen.

Schlagwörter: Lebenserwartung · Sterbetafel · Sterblichkeit · Zensusauswirkungen · Healthy-Migrant-Effekt

Dieser Artikel enthält ergänzende Materialien in einem Online-Anhang: DOI: 10.12765/CPoS2016-06de,

URL: http://www.comparativepopulationstudies.de/index.php/CPoS/article/view/276/248.

Federal Institute for Population Research 2017

(cc) BY-SA
URL: www.comparativepopulationstudies.de DOI: $10.12765 /$ CPoS-2016-05de URN: urn:nbn:de:bib-cpos-2016-05de8 


\section{$1 \quad$ Einleitung}

Die Daten des Zensus 2011 ermöglichen es erstmalig, für Deutschland nach dem derzeit gültigen Gebietsstand demografische Analysen auf Basis von möglichst genauen Bevölkerungsdaten durchzuführen. Da die letzte Volkszählung im früheren Bundesgebiet im Jahr 1987 und in der ehemaligen DDR im Jahr 1981 durchgeführt wurde, mussten sich Analysen für das vereinte Deutschland mit Bezug auf Bevölkerungszahlen bislang auf Daten aus der Bevölkerungsfortschreibung beziehen. Bei der Bevölkerungsfortschreibung wurden die Ergebnisse der letzten Volkszählung im früheren Bundesgebiet sowie ein Auszug des zentralen Registers der DDR vom 03.10.1990 für die neuen Länder unter Berücksichtigung der Statistiken über Geburten und Sterbefälle sowie der Zu- und Fortzüge für einzelne Kalenderjahre fortgeschrieben. Ungenauigkeiten in diesen Statistiken führen zwangsläufig zu Ungenauigkeiten in den fortgeschriebenen Bevölkerungszahlen. Die Qualität der Angaben zu Geburten und Sterbefällen wird dabei als gut eingeschätzt. Der Wanderungssaldo wird dagegen tendenziell zu hoch ausgewiesen, da sich nicht alle Fortziehenden abmelden und deshalb in der Wanderungsstatistik fehlen. Infolgedessen wurde die Einwohnerzahl Deutschlands durch den Zensus 2011 um etwa 1,5 Millionen Personen nach unten korrigiert. Großen Einfluss auf bevölkerungswissenschaftliche Analysen haben zudem Veränderungen der Bevölkerungszahlen in der jeweils zu berücksichtigenden demografischen Untergliederung - beispielsweise nach Alter, Geschlecht oder Staatsangehörigkeit. Im Hinblick auf diese Dimensionen hat es Zensuskorrekturen in unterschiedlichem Ausmaß gegeben (Kaus/Mundil-Schwarz 2015: 29-32).

Der vorliegende Artikel setzt sich mit den Auswirkungen des Zensus 2011 auf die Messung der Lebenserwartung in Deutschland auseinander. Hierfür werden die Daten und die Vorgehensweise der allgemeinen Sterbetafel 2010/12 für Deutschland herangezogen. Eine allgemeine Sterbetafel wird immer dann erstellt, wenn es eine Volkszählung oder einen Zensus gab. Dabei werden allgemeine Sterbetafeln durch Glättung von Zufallseffekten befreit, um die genauen Bevölkerungsdaten zur Offenlegung grundlegender Mortalitätsmuster zu nutzen. Ein besonderer Schwerpunkt wird in diesem Artikel auf die Erstellung von Sterbetafeln nach Staatsangehörigkeit (deutsch/nichtdeutsch) auf Basis der Zensusdaten gelegt. Insbesondere für die ausländische Bevölkerung ließen sich auf Basis der Daten aus der Bevölkerungsfortschreibung mit größer werdendem Abstand zur vorangegangenen Volkszählung keine plausiblen Ergebnisse mehr ermitteln. Verlässliche Kenntnisse zur Sterblichkeit der ausländischen Bevölkerung sind jedoch insbesondere vor dem Hintergrund des steigenden Anteils dieser Bevölkerungsgruppe von hoher Relevanz. Zuwanderung wird häufig als eine Option zur Minderung der demografischen Probleme Deutschlands, dem zu erwartenden Bevölkerungsrückgang und der demografischen Alterung, thematisiert. Kenntnisse zu den Sterblichkeitsverhältnissen der ausländischen Bevölkerung helfen, die damit verbunden Konsequenzen für das Sozialversicherungssystem, insbesondere für die Renten-, Kranken- und Pflegeversicherung, besser abschätzen zu können. Darüber hinaus liefern zuverlässige, nach Staatsangehörigkeit untergliederte, Sterbetafeln eine erste Grundlage für differen- 
zierte Vorausberechnungen der entsprechenden Bevölkerungsgruppen. Zusätzlich tragen Sterblichkeitsparameter wie die Lebenserwartung auch zur Beschreibung der "Lebenslagen" unterschiedlicher Bevölkerungsgruppen bei. Im entsprechenden Wissenschaftsdiskurs ermöglichen es belastbare Ergebnisse auf Basis des Zensus 2011 verschiedene Hypothesen, die in Bezug auf den Healthy-Migrant-Effekt für die ausländische Bevölkerung in Deutschland aufgestellt wurden, neu zu bewerten. Da bei Ergebnissen, die ausschließlich auf Zensusdaten zur Bevölkerung basieren, ein Fortschreibungsfehler zur Erklärung der Befunde ausgeschlossen werden kann, können Erklärungshypothesen mithilfe der vorgelegten Ergebnisse ohne diese Einschränkung diskutiert werden. Einen Überblick zum entsprechenden Stand der Forschung liefert Abschnitt 2 dieses Artikels. In Abschnitt 3 werden die aus diesem Forschungsstand abgeleiteten Forschungsfragen dargelegt und in Abschnitt 4 die verwendeten Daten und Methoden beschrieben. Die Ergebnisse der Analyse werden dann in Abschnitt 5 präsentiert und abschließend in Abschnitt 6 diskutiert.

\section{Stand der Forschung}

Im Fachbericht zur allgemeinen Sterbetafel 2010/12 (Statistisches Bundesamt 2015a) wurde bereits über die zentralen Auswirkungen des Zensus 2011 auf die Erstellung der amtlichen Sterbetafeln und die damit verbunden Konsequenzen für die Messung der Lebenserwartung berichtet. Es wurde aufgezeigt, dass die Lebenserwartung bei Geburt in Deutschland auf Basis des Zensus für Männer um 0,25 Jahre und für Frauen um 0,10 Jahre geringer ist, als wenn sie für den gleichen Zeitraum auf Basis der alten Fortschreibung berechnet worden wäre. Zudem wurde auch auf die Auswirkungen der Berücksichtigung von Zensusdaten auf altersspezifischer Ebene eingegangen. Dort hat sich gezeigt, dass die altersspezifischen Sterbewahrscheinlichkeiten auf Basis des Zensus durchweg höher sind als auf Basis der alten Fortschreibung.

Die am stärksten ausgeprägten Korrekturen der Sterbewahrscheinlichkeiten gab es in den Altersbereichen, in denen auch die Bevölkerung durch den Zensus relativ gesehen am stärksten korrigiert wurde - etwa im Alter 20 bis 40 Jahre und im hohen Alter ab etwa 85 Jahren. Bei Hochbetagten wären die Korrekturen noch deutlicher ausgefallen, wenn nicht ein Extrapolationsverfahren eingesetzt worden wäre. Dieses Verfahren korrigierte die nicht plausiblen Verläufe der Sterbewahrscheinlichkeiten auf Basis der alten Fortschreibung im Alter ab 91 Jahren (Eisenmenger 2003). Die Zensuskorrekturen waren im gesamten Altersbereich bei den Männern ausgeprägter als bei den Frauen (Statistisches Bundesamt 2015a: 21-23). Im Hinblick auf Zensusauswirkungen für eine separate Berechnung von Sterbetafeln für die deutsche und die ausländische Bevölkerung wurden bislang Ergebnisse unter Verwendung der vorläufigen Bevölkerungsfortschreibung auf Basis des Zensus 2011 für das Jahr 2013 vorgelegt. Demnach geht die Differenz zwischen Nichtdeutschen und Deutschen in der Lebenserwartung bei Geburt für Männer von 4,8 auf 2,6 Jahre zurück, bei Frauen wird eine zensuskorrigierte Differenz von 1,7 Jahren zugunsten der Ausländerinnen festgestellt (Koh/s 2015: 522). 
Die hinter den Zensuskorrekturen der Sterblichkeitsmuster stehenden Korrekturen der Bevölkerung nach Alter und Geschlecht durch den Zensus 2011 sind in Kaus/ Mundil-Schwarz (2015) zum Stichtag 31.12.2011 dargelegt. An selber Stelle wurde auch herausgearbeitet, dass die Korrekturen bei der ausländischen Bevölkerung erheblich größer waren als bei der deutschen Bevölkerung. Bei einem Korrekturfaktor von 1,9\% für die Gesamtbevölkerung wurde die deutsche Bevölkerung nur um $0,6 \%$ korrigiert, die ausländische Bevölkerung jedoch um 14,4 \%. Die Korrekturen bei der ausländischen Bevölkerung betreffen dabei breitere Altersbereiche als bei der Gesamtbevölkerung. Auch bei der ausländischen Bevölkerung wurde die männliche Bevölkerung stärker korrigiert als die weibliche (Kaus/Mundil-Schwarz 2015: 30).

Eine deutliche Korrektur der ausländischen Bevölkerung durch den Zensus war bereits im Vorfeld zu erwarten. Ein Grund hierfür waren die nicht plausiblen Mortalitätsmuster, die man auf Basis der alten Fortschreibung für die ausländische Bevölkerung ermittelt hat. Mit den Daten der Bevölkerungsfortschreibung fanden Forscher stets ausgeprägte Unterschiede in den Überlebensverhältnissen von Nichtdeutschen im Vergleich zur deutschen Bevölkerung bzw. der Allgemeinbevölkerung zugunsten der nichtdeutschen Personen. Ein Großteil dieses Effekts wurde dabei mit Datenartefakten wie einer überhöhten ausländischen Bevölkerung infolge eines Fortschreibungsfehlers bzw. der Nichtberücksichtigung von Sterbefällen im Ausland erklärt (Luy 2007; Kibele et al. 2008; Kohls 2008a/b, 2011, 2012; Mammey/ Schwarz 2002; Robert Koch Institut/Statistisches Bundesamt 2008). Als Konsequenz wurden verschiedene andere Datenquellen herangezogen, um die Überlebensverhältnisse der ausländischen Bevölkerung in Deutschland zu quantifizieren und Unterschiede zur deutschen Bevölkerung bzw. der Allgemeinbevölkerung aufzuzeigen.

Luy (2007) hat beispielsweise die Daten des Integrationssurveys des Bundesinstituts für Bevölkerungsforschung genutzt, um mit einer indirekten Methode (der Orphanhood Methode in Kombination mit dem Logit-Brass-Modell) die Lebenserwartung von ausländischen Personen im Alter 35 zu schätzen. Im Ergebnis zeigen sich viel kleinere Sterblichkeitsunterschiede als auf Basis der Bevölkerungsfortschreibung. Eine geringere Sterblichkeit der ausländischen Bevölkerung findet sich jedoch auch mit dieser indirekten Herangehensweise. Zu ähnlichen Ergebnissen kommt Kohls (2008b, 2011, 2012) mit Daten des Ausländerzentralregisters (AZR). Misst man die Lebenserwartung bei Geburt der ausländischen Bevölkerung mithilfe des AZR, so sind die Differenzen zur deutschen Bevölkerung beispielsweise im Jahr 2009 um etwa 0,8 (Frauen) bis 1,0 Jahre (Männer) geringer, als wenn diese mit Daten der Bevölkerungsfortschreibung berechnet werden. Vor 2005 fand man auch mit den Daten des AZR deutlichere Unterschiede zwischen der deutschen und der ausländischen Bevölkerung, die sich jedoch infolge einer Registerbereinigung im Jahr 2004 erheblich reduziert haben (Koh/s 2012: 149; Opfermann et al. 2006: 484-485).

Mit Daten der gesetzlichen Rentenversicherung (GRV) fand Koh/s (2008b) ebenfalls den Effekt einer höheren Lebenserwartung der ausländischen Bevölkerung in Bezug auf die fernere Lebenserwartung im Alter 60. Die Differenzen sind zwar kleiner als auf Basis der Fortschreibung des Bevölkerungsbestandes, ein höherer Wert der ausländischen Bevölkerung im Vergleich zur deutschen Bevölkerung bleibt jedoch bestehen. Die Sterblichkeitsunterschiede zwischen deutscher und ausländi- 
scher Bevölkerung gehen den Ergebnissen zufolge zwischen 1994 und 2006 stark zurück (Koh/s 2008b: 32-38). Bei weiteren Analysen mit Daten der GRV zeigt sich sogar eine höhere Sterblichkeit der ausländischen Bevölkerung über 60 Jahre im Vergleich zur deutschen Bevölkerung seit 2002 (Koh/s 2008a, 2010, 2011, 2012). Im Alter zwischen 20 und 59 lässt sich auch mit Daten der GRV durchweg eine geringere Sterblichkeit der ausländischen Bevölkerung im Vergleich zur deutschen Bevölkerung ausweisen (Koh/s 2010, 2012). Die Säuglings-, Kinder- und Jugendsterblichkeit der ausländischen Bevölkerung ist tendenziell höher als die der deutschen Bevölkerung (Koh/s 2011, 2012). Hier gilt die Bevölkerungsfortschreibung als verlässlich, weil die in höherem Alter relevanten Verzerrungen in diesem Alter "praktisch nicht bestehen" (Koh/s 2008c: 41).

Die Vorteile in den Überlebensverhältnissen der ausländischen Bevölkerung werden dabei überwiegend auf einen sogenannten Healthy-Migrant-Effekt zurückgeführt. Dieser besagt, dass wandernde Personen positiv selektiert, d.h. besonders gesund im Vergleich zur nicht wandernden Bevölkerung sind und folglich eine geringere Sterblichkeit haben. Das Postulieren eines solchen Effektes suggeriert jedoch auch, dass der Sterblichkeitsvorteil mit steigender Aufenthaltsdauer abnehmen sollte und sich insbesondere bei vorhandenen sozioökonomischen Benachteiligungen der ausländischen Bevölkerung in einen Sterblichkeitsnachteil wandeln kann (Razum 2009: 268-269; Koh/s 2008c: 17). Zahlreiche Hinweise für eine deutlich verringerte Gesamtsterblichkeit von wandernden Personen finden sich durchweg nicht nur in den bereits erwähnten nationalen Studien für Deutschland, sondern auch in zahlreichen internationalen Studien für andere Länder (Koh/s 2012: 69-70). Die meisten Studien vergleichen dabei für ihre Befunde zum Healthy-Migrant-Effekt die Gesundheit bzw. Sterblichkeit der einheimischen mit der jeweils zugewanderten Bevölkerung. Kennedy und Kidd (2015) zeigen gleich für vier verschiedene große Einwanderungsländer (USA, Kanada, Großbritannien und Australien), dass Zuwanderer auch im Vergleich zu den Einheimischen des jeweiligen Herkunftslandes typischerweise deutliche Gesundheitsvorteile aufweisen. Dennoch kann nicht von einem universellen Muster gesprochen werden. Betrachtet man den selbst berichteten Gesundheitszustand, so lässt sich für mehrere europäische Länder zeigen, dass Zuwanderer einen schlechteren Gesundheitszustand aufweisen als die jeweilige einheimische Bevölkerung (siehe Nielsen und Krasnik (2010) für eine ausführliche Review der vorliegenden Studien). Moullan und Jusot (2014) finden auf Basis harmonisierter Daten von Gesundheitssurveys Hinweise für einen Gesundheitsvorteil von Migranten in Italien, jedoch eine schlechtere Gesundheit von Zugewanderten in Frankreich, Belgien und Spanien. Die Autoren führen diese Befunde auf unterschiedlich wirkende Selektionseffekte bei der Einwanderung und bei der Integration von Migranten zurück. Der Gesundheitsvorteil von Migranten in Italien wird unter anderem auch damit begründet, dass die Einheimischen dort in ihrer Selbsteinschätzung einen schlechteren Gesundheitszustand aufweisen als beispielsweise in Frankreich (Moullan/Jusot 2014: 84).

Im deutschen Kontext ordnet Koh/s $(2011,2012)$ die Sterblichkeitsunterschiede zwischen der deutschen und der ausländischen Bevölkerung folgendermaßen ein: Ein Sterblichkeitsvorteil in den mittleren Altersgruppen ist bei der ausländischen 
Bevölkerung zweifelsfrei nachweisbar und kann mit den typischen Selektionseffekten erklärt werden. Der überwiegende Anteil der arbeitsmarktorientierten Migranten wandert in einem Alter zwischen 25 und 40 Jahren zu. Entsprechend weist ein hoher Anteil der ausländischen Bevölkerung im Erwerbsalter eine vergleichsweise geringe bisherige Aufenthaltsdauer auf, weswegen Selektionseffekte, die direkt nach der Zuwanderung am stärksten wirken, für eine niedrigere Sterblichkeit der ausländischen Bevölkerung sorgen.

Die geringeren Sterblichkeitsunterschiede bzw. eine höhere Sterblichkeit der ausländischen Bevölkerung etwa ab Alter 65 erklären sich durch Adaptionseffekte. Im Zuge einer längeren Aufenthaltsdauer nähern sich die Sterblichkeitsverhältnisse von deutscher und ausländischer Bevölkerung an und können bei gegebenen sterblichkeitsrelevanten Benachteiligungen der ausländischen Bevölkerung auch in eine höhere Sterblichkeit bei Nichtdeutschen umschlagen. Die vergleichsweise hohen relativen Sterblichkeitsrisiken der ausländischen Bevölkerung im Alter von 65 bis 69 Jahren können beispielsweise durch den hohen Anteil von ehemaligen Gastarbeitern in dieser Altersgruppe erklärt werden. Diese wanderten im Laufe der 1950er bis 1970 er Jahre ein und waren in der Regel über Jahrzehnte stark belastenden Arbeitsbedingungen ausgesetzt, wodurch die relativ hohe Sterblichkeit der entsprechenden Altersgruppe begründet sein kann (Koh/s 2012: 181).

Die Ursachen für die höhere Sterblichkeit von Säuglingen, Kindern und Jugendlichen unter der ausländischen Bevölkerung sind bislang kaum theoretisch begründet worden. Weber et al. (1990) haben empirisch aufgezeigt, dass die erhöhte Säuglingssterblichkeit der mit ausländischer Staatsbürgerschaft in Deutschland geborenen Kinder vorwiegend durch eine im Vergleich zu deutschen Müttern verkürzte Schwangerschaftsdauer sowie durch ein erhöhtes Auftreten von Fehlbildungen und Infektionskrankheiten begründet ist. Dies könnte die Folge einer unzureichenden Betreuung schwangerer Migrantinnen sein. Bei Kindern und Jugendlichen haben Weber et al. (1990) die höhere Sterblichkeit durch Unfälle als Haupterklärungsfaktor für die höhere Sterblichkeit der ausländischen Bevölkerung identifiziert. Bei anderen Todesursachen wiesen ausländische und deutsche Kinder bzw. Jugendliche sehr ähnliche Sterblichkeitsrisiken auf. Mit neueren Daten lassen sich Unterschiede in der Unfallsterblichkeit zwischen der deutschen und der ausländischen Bevölkerung bei Kindern und Jugendlichen allerdings nicht mehr nachweisen. Ausländische Kinder und Jugendliche wiesen zwischen 1999 und 2004 sogar eine etwas niedrigere Unfallsterblichkeit im Vergleich zu gleichaltrigen Deutschen auf. Lediglich in der Verteilung der Unfallarten konnten noch gewisse Unterschiede zwischen beiden Gruppen nachgewiesen werden (Robert Koch Institut/Statistisches Bundesamt 2008: 71-73).

\section{Forschungsfragen}

Die Auswirkungen des Zensus auf die Erstellung der amtlichen Sterbetafeln für den Zeitraum 2010/12 wurden bereits an anderer Stelle thematisiert (Statistisches Bundesamt 2015a). Dabei wurde ein Fokus auf die Auswirkungen des Zensus auf die 
Berechnung der altersspezifischen Sterbewahrscheinlichkeiten gelegt. Auf Ebene der altersspezifischen Lebenserwartung wurde der Zensuseffekt bislang nur für die Lebenserwartung Neugeborener quantifiziert. Im vorliegenden Artikel sollen diese Effekte für den gesamten Altersverlauf analysiert werden. Die erste Forschungsfrage dieser Arbeit lautet demnach:

Welche Auswirkungen haben die Zensuskorrekturen der Bevölkerung auf die altersspezifische Lebenserwartung in Deutschland und wie lassen sich diese Korrekturen im Kontext der jährlichen Sterblichkeitsveränderungen einordnen?

Besonders starke Auswirkungen durch die Zensuskorrekturen werden im Hinblick auf die Erstellung von Sterbetafeln für die ausländische Bevölkerung erwartet. Die Schwächen der amtlichen Statistik in Bezug auf die Analyse von Mortalitätsmustern der ausländischen Bevölkerung wurden in den vorhandenen Publikationen zum Thema im Wesentlichen auf den durch unterlassene Abmeldungen entstandenen Fortschreibungsfehler seit den letzten Volkszählungen im früheren Bundesgebiet und der (ehemaligen) DDR zurückgeführt. Der vorliegende Artikel widmet sich deshalb einer zentralen zweiten Forschungsfrage:

Wie hoch sind die Sterblichkeitsunterschiede zwischen deutscher und ausländischer Bevölkerung ohne Fortschreibungsfehler auf Basis der Daten des Zensus 2011?

Insbesondere bei der Berechnung einer Sterbetafel für die ausländische Bevölkerung sind methodische Aspekte nicht trivial. Eine Extrapolation von Sterbewahrscheinlichkeiten im hohen Alter kann erhebliche Auswirkungen auf die tatsächlich gemessene Höhe der Lebenserwartung haben. Deswegen sollen im Kontext der Beantwortung der zweiten Forschungsfrage verschiedene methodische Aspekte in ihrer Bedeutung hervorgehoben werden. So sollen unter anderem die Fragen geklärt werden, bis zu welchem Alter die Daten des Zensus 2011 eine Ermittlung von plausiblen Mustern der altersspezifischen Mortalität erlauben und welche Verbesserungen es diesbezüglich im Vergleich zu den Fortschreibungsdaten gibt. Die Beantwortung der zweiten Forschungsfrage kann somit Aufschluss darüber geben, in welcher Gewichtung die in der Literatur angeführten Erklärungsfaktoren (Selektions- und Adaptionshypothese) tatsächlich zu den Sterblichkeitsunterschieden von deutscher und ausländischer Bevölkerung beitragen. Lässt sich mithilfe der als verlässlich geltenden Zensusdaten nachweisen, dass sich die Sterblichkeitsunterschiede zwischen der deutschen und der ausländischen Bevölkerung mit dem Alter stark angleichen, wäre ein deutlicher Hinweis für ein Wirken der Adaptionshypothese erbracht. Unterscheiden sich die Sterbewahrscheinlichkeiten beider Bevölkerungsgruppen bis ins hohe Alter systematisch zugunsten der ausländischen Bevölkerung, würde dies für die Selektionshypothese sprechen. Lassen sich auf Basis der Zensusdaten keine Unterschiede zwischen deutscher und ausländischer Bevölkerung finden, könnten die bislang gefundenen Unterschiede komplett auf Ungenauigkeiten in der Bevölkerungsfortschreibung zurückgeführt werden und das Anführen der Hypothesen zur Erklärung der Unterschiede wäre hinfällig. 


\section{Daten und Methoden}

\subsection{Daten}

Alle Berechnungen wurden mit den Daten der amtlichen Statistik zur Bevölkerung nach Geschlecht und Geburtsjahr und zu den Gestorbenen nach Geschlecht, Alter und Geburtsjahr durchgeführt. Als Bevölkerung zum Zensusstichtag 2011 dienen dabei die Ausgangsdaten der Bevölkerungsfortschreibung aus dem Zensus. Die Statistischen Ämter des Bundes und der Länder haben dazu die Berechnung der aus dem Zensus 2011 resultierenden Ausgangsdaten für die Bevölkerungsfortschreibung optimiert, auch im Hinblick auf die Berechnung demografischer Maße (Statistisches Bundesamt 2015c). Diese Daten und die darauf aufbauende endgültige Bevölkerungsfortschreibung sind deshalb für Sterblichkeitsberechnungen adäquat. Auch im Vergleich mit alternativen Datenquellen zur Ermittlung von Sterblichkeitsverhältnissen der ausländischen Bevölkerung wie dem AZR oder den Daten der GRV haben die Zensusdaten zahlreiche Vorteile. Das AZR hat zwar prinzipiell ebenfalls die vollständige Erfassung der ausländischen Bevölkerung zum Ziel, jedoch sind ähnlich wie bei den Daten der Bevölkerungsfortschreibung immer wieder Bereinigungen notwendig, da insbesondere Abgänge nicht vollständig erfasst werden können (Fleischer 1989; Opfermann et al. 2006). Die letzte umfassende Bereinigung des AZR hat in den Jahren 2000 bis 2004 stattgefunden, sodass gegenwärtig wahrscheinlich erneuter Bereinigungsbedarf besteht. Zudem ist die Meldung von Säuglingssterbefällen im Ausländerzentralregister nicht verpflichtend (Koh/s 2012: 148), was die Ermittlung der wichtigen Komponente Säuglingssterblichkeit auf Basis dieser Datenquelle erschwert. Unterschiedliche Melde- und Berichtswege sowie unterschiedliche Definitionen im Hinblick auf den Aufenthalt der Ausländerinnen und Ausländer zur Pflege des Ausländerzentralregisters erschweren zudem den Vergleich zu den auf üblichem Wege ermittelten Daten für die deutsche Bevölkerung (Opfermann et al. 2006; Kaus/Mundil-Schwarz 2015). Analysen auf Basis der Daten der GRV sind nicht repräsentativ für die Gesamtbevölkerung, da nur die aktiv versicherten Personen berücksichtigt werden können und beispielsweise Selbständige oder Beamte ausgeschlossen sind und somit keine Vollerhebung der Bevölkerung vorliegt (Koh/s 2008c: 33). Auch die Vergleichbarkeit der Ergebnisse zwischen den Geschlechtern ist durch die geringere Erwerbsbeteiligung der Frauen eingeschränkt. Die Säuglings-, Kinder und Jugendsterblichkeit lässt sich mit Daten der gesetzlichen Rentenversicherung ebenfalls nicht und die Erwachsenensterblichkeit vor Renteneintritt nur mit Einschränkungen quantifizieren, weswegen auf Basis dieser Daten keine vollständigen Sterbetafeln aufgestellt werden können.

\subsection{Methodische Überlegungen}

Die in diesem Artikel durchgeführten Analysen basieren auf der vom Statistischen Bundesamt zur Erstellung der allgemeinen Sterbetafel 2010/12 angewandten Methodik (Statistisches Bundesamt 2015a). Zentraler Ansatzpunkt zur Erstellung der allgemeinen Sterbetafel 2010/12 war es, die altersspezifischen Sterbewahrschein- 
lichkeiten mit der Geburtsjahrmethode nach Becker (1874) und Zeuner (1869) unter vollständiger Berücksichtigung der Zensusbestände zu berechnen. Der Einfluss von Wanderungen im Betrachtungszeitraum wurde dabei unter Zuhilfenahme der sogenannten Wittstein-Balducci-Hypothese berücksichtigt (Wittstein 1862; Balducci 1917). Der Vorteil dieses methodischen Ansatzes liegt darin, dass zur Berechnung der Sterbewahrscheinlichkeiten für einen Dreijahreszeitraum nicht auf Daten aus der Bevölkerungsfortschreibung zurückgegriffen werden muss, sondern die möglichst genauen Bevölkerungszahlen des Zensus vollständig genutzt werden können. Der Einfluss von Fortschreibungs- oder Rückrechnungsfehlern - beispielsweise verursacht durch unterlassene Abmeldungen von Fortzügen im relevanten Zeitraum - kann somit ausgeschlossen werden.

Mit der Herangehensweise des Statistischen Bundesamtes für die allgemeine Sterbetafel 2010/12 wurden für diesen Artikel separat Sterbetafeln nach Staatsangehörigkeit für die deutsche und für die ausländische Bevölkerung erstellt. Dabei gilt es verschiedene methodische Aspekte zu berücksichtigen:

Die Ergebnisse einer Periodensterbetafel bilden grundsätzlich die Überlebensverhältnisse einer hypothetischen Kohorte ab. Im Hinblick auf die Lebenserwartung bei Geburt liefern Periodensterbetafeln somit die Antwort auf die Frage, wie lange Personen eines bestimmten Alters noch leben würden, wenn sie dauerhaft den Überlebensverhältnissen des Betrachtungszeitraums ausgesetzt wären. Berechnet man eine Periodensterbetafel für Untergruppen einer Gesamtbevölkerung, gilt es die Annahme zu hinterfragen, ob die gemessenen altersspezifischen Sterbewahrscheinlichkeiten dieser Untergruppe tatsächlich repräsentativ für das jeweilige Alter der Personengruppe sind. Bei einer Sterbetafel für die ausländische Bevölkerung bedeutet dies, dass ein großer Anteil der in der Berechnung berücksichtigten Bevölkerung erst im Laufe des Lebens zugewandert ist. Für diese Personengruppe liegen somit keine Informationen zur Sterblichkeit vor der Zuwanderung vor. Folglich spielt bei der Aufstellung von Sterbetafeln für die ausländische Bevölkerung zusätzlich zur Annahme einer hypothetischen Kohorte eine weitere Annahme eine wichtige Rolle: Nämlich, dass die (unbeobachtete) Sterblichkeit vor der Zuwanderung mit der beobachteten Sterblichkeit im Inland von Personen entsprechenden Alters, die bereits in früherem Alter zugewandert oder mit einer ausländischen Staatsbürgerschaft geboren wurden, identisch sei. Deshalb sollte die (Perioden-)Lebenserwartung der ausländischen Bevölkerung als durchschnittliche Lebensdauer von Personen interpretiert werden, die dauerhaft den im Inland herrschenden Überlebensverhältnissen der ausländischen Bevölkerung im Betrachtungszeitraum unterworfen wären. Sterblichkeitsverhältnisse vor der Zuwanderung können dabei nicht berücksichtigt werden. Die notwendige zusätzliche Annahme betrifft insbesondere die Lebenserwartung bei Geburt und die fernere Lebenserwartung in jungen Altersjahren. Die fernere Lebenserwartung in höherem Alter, beispielsweise ab Alter 60, dürfte davon weniger betroffen sein, da Wanderungsbewegungen in diesem Altersbereich nur noch eine untergeordnete Rolle spielen. Dass sich die Zusammensetzung der ausländischen Bevölkerung durch sich verändernde Wanderungsmuster deutlich schneller ändert als die der deutschen Bevölkerung, sorgt zusätzlich dafür, dass die Annahme einer hypothetischen Kohorte problematischer ist als bei der Aufstel- 
lung einer Periodensterbetafel für die Gesamtbevölkerung (in der die ausländische Bevölkerung nur einen kleinen Anteil hat) oder für die Bevölkerung mit deutscher Staatsangehörigkeit.

Ein weiterer Punkt, den es zu bedenken gilt, sind die Auswirkungen eines sogenannten "unhealthy re-migration effects" (Razum et al. 1998: 302). Damit gemeint ist die möglicherweise selektive Rückwanderung (etwa zu Behandlungszwecken) von ausländischen Personen mit einer schweren Krankheit, die nach ihrer Ausreise im Ausland sterben (Koh/s 2008c: 21). Auch Sterbefälle der Wohnbevölkerung, die aus anderen Gründen im Ausland stattfinden (z.B. im Urlaub), können sich verzerrend auswirken, wenn die entsprechenden Meldungen die statistischen Ämter nicht erreichen. Die Sterbewahrscheinlichkeiten werden dann durch Effekte auf den Zähler (nicht gemeldete Sterbefälle) bzw. den Nenner (unterlassene Abmeldung eines Fortzugs) beeinflusst. Für die Jahre 2011 und 2012 sind jeweils etwas über 3.500 Fälle von Personen mit Wohnsitz in Deutschland bekannt, die im Ausland starben. ${ }^{1}$ Bei den deutschen Standesämtern wurden in beiden Jahren etwas unter 1.000 von diesen Sterbefällen aus dem Ausland gemeldet und konnten somit in der Statistik der Sterbefälle berücksichtigt werden. Demnach verbleibt eine Lücke von etwa 2.500 Sterbefällen jährlich, die im europäischen Ausland passieren, in der Sterbestatistik jedoch keine Berücksichtigung finden (was einem Anteil von etwa 0,3\% an allen Sterbefällen entspricht). Unbekannt bleibt, wie viele Sterbefälle es darüber hinaus im Ausland gibt, etwa von Personen, die sowohl in Deutschland als auch im Ausland zur Bevölkerung zählen.

Eine zusätzliche datenbedingte Einschränkung besteht darin, dass Wechsel von Staatsangehörigkeiten bei einer separaten Erstellung von Sterbetafeln für Deutsche und Ausländer nicht berücksichtigt werden können. Wenn beispielsweise eine Person zum Zeitpunkt des Zensus eine ausländische Staatsangehörigkeit besaß, nach dem Zensus die Staatsangehörigkeit gewechselt hat und danach gestorben ist, dann wurde der Sterbefall als Sterbefall der deutschen Bevölkerung registriert und ist als solcher - in Bezug auf die deutsche Bevölkerung am Zensusstichtag - in die Berechnung eingegangen. Unter Berücksichtigung einer Einbürgerungsquote von 1,4 bis $1,7 \%$ im relevanten Zeitraum (Statistisches Bundesamt 2015b) und der Tatsache, dass die meisten eingebürgerten Personen den Betrachtungszeitraum überleben, dürfte ein eventuell verzerrender Effekt allerdings vernachlässigbar sein.

Auch die Anwendung des lus-soli-Prinzips seit dem Jahr 2000 (nach dem in Deutschland geborene Kinder von ausländischen Eltern unter gewissen Voraussetzungen die deutsche Staatsbürgerschaft bekommen), kann sich verzerrend auf eine

1 Basierend auf unveröffentlichten Auswertungen des Statistischen Bundesamtes (Tabelle N30) und der Tabelle zu "Todesursachen - absolute Zahl im Berichtsland und für Einwohner" (hlth cd_aro) der Online-Datenbank von Eurostat (2015). In der Eurostat-Tabelle werden „alle Todesfälle von Einwohnern in oder außerhalb ihres Heimatlandes" nachgewiesen. Zieht man von den in Deutschland registrierten Sterbefällen diejenigen ab, die aus dem Ausland gemeldet wurden, erhält man die Sterbefälle im Inland. Die Differenz zwischen dieser Zahl und der Zahl von Eurostat zu allen Todesfällen in oder außerhalb von Deutschland betrug in 2011 und 2012 jeweils etwa 3.500 Fälle. Sterbefälle im nichteuropäischen Ausland bleiben dabei unberücksichtigt. 
nach Staatsangehörigkeit getrennte Sterbetafelberechnung auswirken. Insbesondere bei der direkt aus der Geburtenstatistik berechneten Säuglingssterblichkeit kann es zu Verzerrungen kommen, wenn eine Geburt mit ausländischer Staatsbürgerschaft registriert wird, eine spätere Feststellung der deutschen Staatsbürgerschaft diese Statistik jedoch nicht mehr beeinflusst. Ein etwaiger Sterbefall eines deutschen Kindes würde in diesem Beispiel auf die falsche Bevölkerung bezogen werden. Diese Konstellation würde somit für eine Unterschätzung der Säuglingssterblichkeit bei der ausländischen Bevölkerung und eine - aller Wahrscheinlichkeit marginale - Überschätzung bei der deutschen Bevölkerung sorgen. Bei einer Berechnung der Sterbewahrscheinlichkeiten auf Basis der Zensusdaten dürfte es in den folgenden Altersjahren keine Verzerrung infolge der Anwendung des lussoli-Prinzips geben, da die entsprechenden Fälle im Rahmen des Zensus korrigiert wurden (Kaus/Mundil-Schwarz 2015: 31). Bei einer Berechnung auf Basis der Fortschreibungsdaten vor dem Zensus 2011 wäre der Effekt auch bei der Berechnung für Sterbewahrscheinlichkeiten in den folgenden Altersstufen relevant.

Um die rohen altersspezifischen Sterbewahrscheinlichkeiten zu glätten, wurde für die allgemeine Sterbetafel 2010/12 des Statistischen Bundesamtes ein P-SplineAnsatz nach Eilers und Marx (1996) gewählt, der durch die Berücksichtigung der Binomialverteilung speziell auf die Glättung von Sterbewahrscheinlichkeiten zugeschnitten ist (zur Nieden et al. 2016: 65). Eine Glättung von Sterbewahrscheinlichkeiten ist insbesondere dann notwendig, wenn generelle Muster ohne Zufallseinflüsse oder Sondereffekte abgeleitet werden sollen. Solche generellen altersspezifischen Muster eignen sich beispielsweise in besonderem Maße als Basis für eine Vorausberechnung der Sterblichkeit, da Zufallseinflüsse somit nicht dauerhaft fortgeschrieben werden und die Ableitung zukünftiger Mortalitätsmuster plausibler ist. Bei den zusätzlich für diesen Aufsatz durchgeführten Berechnungen von Sterbetafeln nach Staatsangehörigkeit wurden die zugrundeliegenden altersspezifischen Sterbewahrscheinlichkeiten ebenfalls mit dem P-Spline-Ansatz geglättet, um eine Überinterpretation von zufälligen Überschneidungen auszuschließen und sich auf die Analyse von grundlegenden Mustern zu beschränken.

Damit auf Basis dieser grundlegenden Muster Aussagen darüber getroffen werden können, in welchen Altersbereichen sich die Sterbewahrscheinlichkeiten von Deutschen und Nichtdeutschen signifikant unterscheiden, wurden zusätzlich Konfidenzintervalle für die altersspezifischen Sterbewahrscheinlichkeiten berechnet. Hierfür wurde angenommen, dass die jeweils beobachtete altersspezifische Sterbewahrscheinlichkeit $q(x)$ den wahren Erwartungswert abbildet und errechnet, in welchen Grenzen Zufallsstichproben mit einer 95 \%igen Wahrscheinlichkeit liegen würden (Salzmann/Koh/s 2006: 187-188).

Wie bei der allgemeinen Sterbetafel 2010/12 wurden auch bei den für diesen Aufsatz zusätzlich erstellten Sterbetafeln die Sterbewahrscheinlichkeiten im hohen Alter bei einsetzenden unplausiblen Verläufen extrapoliert. Nicht plausible Verläufe können dabei sich überschneidende Sterbewahrscheinlichkeiten von Männern und Frauen und/oder eine deutliche Abflachung bzw. ein Absinken der Sterbewahrscheinlichkeiten sein. Zum Einsatz als Extrapolationsverfahren kam hierbei das lo- 
gistische Modell, welches auf Arbeiten von Perks (1932) zurückgeht und in Thatcher et al. (1998) als für diesen Zweck besonders geeignet bewertet wurde.

Um die Effekte des Zensus herauszuarbeiten, wurden auch Sterbetafeln mit Daten aus der Bevölkerungsfortschreibung auf Grundlage der Volkszählung 1987 im früheren Bundesgebiet bzw. des Auszugs des zentralen Registers der DDR vom 03.10.1990 in den neuen Ländern (der alten Fortschreibung vor Zensus) durchgeführt (Statistisches Bundesamt 2013). Zudem wurde auch die amtliche Sterbetafel 2009/11 herangezogen (Statistisches Bundesamt 2012), um den Zensuseffekt auf die Erstellung der allgemeinen Sterbetafel 2010/12 mit dem Rückgang der Sterblichkeit zur vorangegangen Sterbetafel in Kontext stellen zu können. Hierfür wurden mithilfe der Differenzen in der altersspezifischen Lebenserwartung e $(x)$ aus der Sterbetafel 2009/11 und einer Sterbetafel für 2010/12 auf Basis der alten Fortschreibung und auf Basis des Zensus jeweils ein Sterblichkeitseffekt (SE)

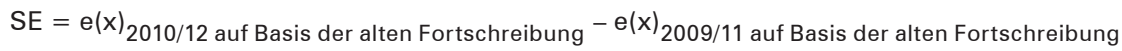

und ein Zensuseffekt (ZE)

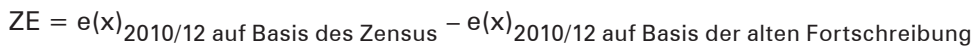

berechnet. Die Summe der beiden Effekte bildet die tatsächliche Veränderung in der altersspezifischen Lebenserwartung zwischen der Sterbetafel für 2009/11 und der allgemeinen Sterbetafel 2010/12 ab.

Um die Unterschiede in der Sterblichkeit von Deutschen und Nichtdeutschen herausarbeiten zu können wurde eine relative Maßzahl $\left(U_{x}\right)$ berechnet, die die Differenz der altersspezifischen Sterbewahrscheinlichkeiten $\left(q_{x}\right)$ der beiden Gruppen ins Verhältnis zu den Werten für die deutsche Bevölkerung setzt:

$$
U_{x}=\frac{q_{x, \text { Nichtdeutsche }}-q_{x, \text { Deutsche }}}{q_{x, \text { Deutsche }}}
$$

\section{Analysen}

\subsection{Auswirkungen des Zensus auf die Sterblichkeitsmessung der Gesamtbevölkerung}

Die Lebenserwartung bei Geburt in Deutschland betrug im Zeitraum 2010/12 laut der allgemeinen Sterbetafel 77,7 Jahre für Männer und 82,8 Jahre für Frauen. Im Gegensatz zur vorangegangen Sterbetafel 2009/11 sind die Werte für die Lebenserwartung bei Geburt somit bei den Männern unverändert und bei den Frauen nur leicht verändert (+ 0,1 Jahre). In Abbildung 1 sind die Unterschiede zwischen der Sterbetafel 2009/11 und der allgemeinen Sterbetafel 2010/12 separiert nach einem Zensus-, einem Sterblichkeits- und einem Gesamteffekt für den gesamten Altersverlauf der (ferneren) Lebenserwartung dargestellt. Bei den Männern wie auch bei den Frauen wäre die Lebenserwartung bei Geburt sowie die fernere Lebenserwartung bis etwa Alter $70 \mathrm{im}$ Vergleich der beiden Sterbetafeln ausgehend von früheren Zählungen um etwa 0,2 Jahre angestiegen, wenn es keinen Zensus gegeben 
Abb. 1: Differenzen in der altersspezifischen Lebenserwartung zwischen der Sterbetafel 2009/11 und der allgemeinen Sterbetafel 2010/12 nach Einzeleffekten für Männer und Frauen

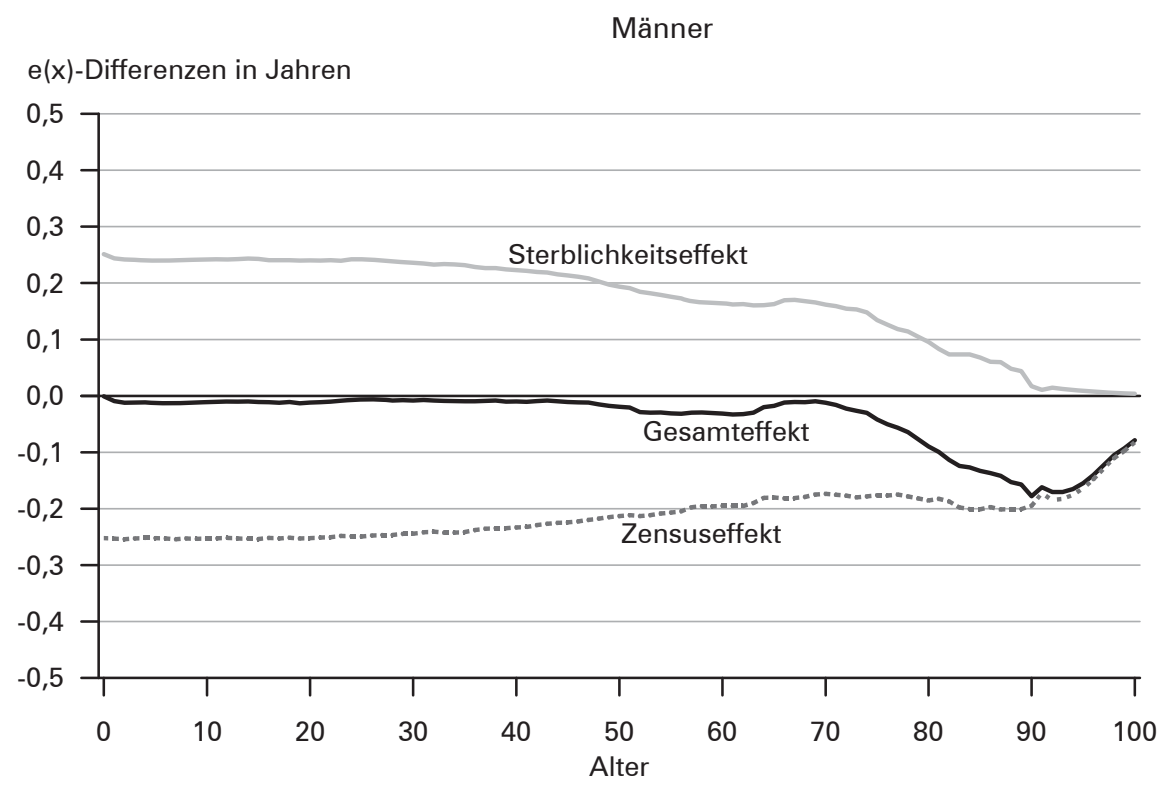

Frauen

e(x)-Differenzen in Jahren

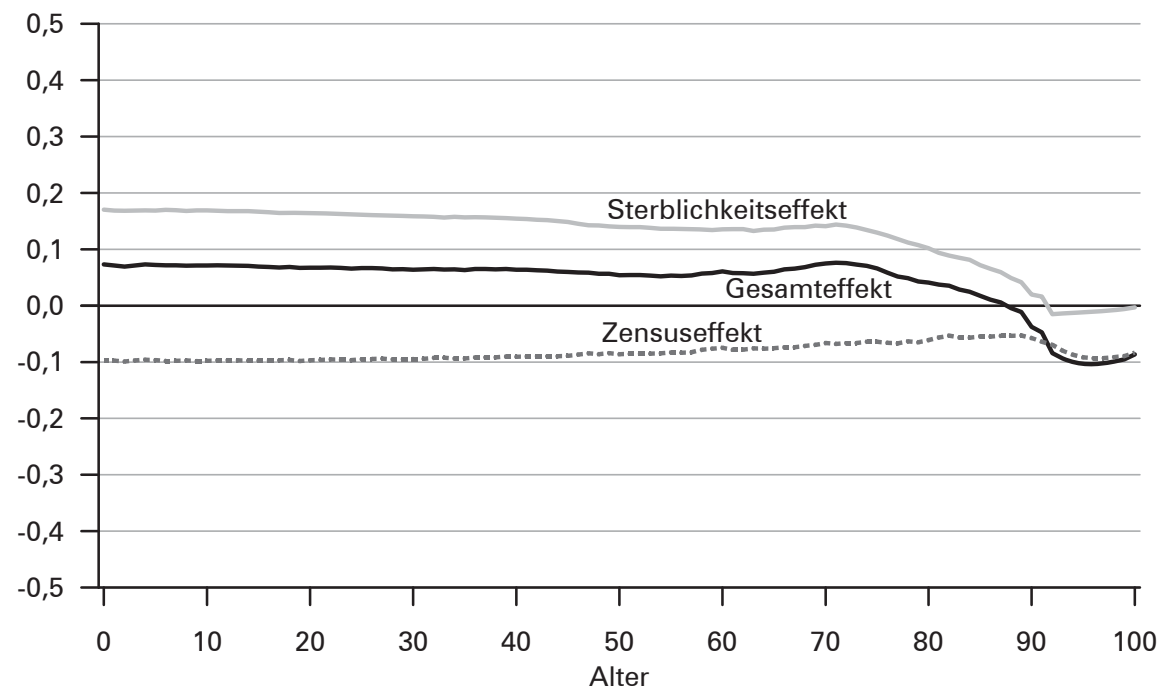

Quelle: Statistisches Bundesamt, eigene Darstellung und Berechnung 
hätte (Sterblichkeitseffekt). Der Rückgang der Sterblichkeit und somit der Anstieg der Lebenserwartung wäre bei den Männern dabei etwas ausgeprägter gewesen als bei den Frauen. Bei beiden Geschlechtern gehen die Gewinne an verbleibender Lebenserwartung durch den Sterblichkeitseffekt spätestens ab Alter 70 bis Alter 90 kontinuierlich zurück. Oberhalb von Alter 90 ist praktisch keine Veränderung in der ferneren Lebenserwartung auf Basis der alten Fortschreibungsdaten messbar. Dabei muss berücksichtigt werden, dass zur Berechnung des Effekts ab Alter 91 jeweils Werte verglichen werden, die aus der Anwendung eines Extrapolationsmodells resultieren. Der vor Alter 90 beobachtete Rückgang des Sterblichkeitseffekts deutet jedoch darauf hin, dass der ausbleibende Anstieg der ferneren Lebenserwartung oberhalb von Alter 90 durchaus plausibel ist.

Die Zensuskorrekturen bei der Erstellung der allgemeinen Sterbetafel sorgen dafür, dass die fernere Lebenserwartung im Zeitraum 2010/12 im gesamten Altersverlauf bei beiden Geschlechtern geringer ist als auf Basis der alten Fortschreibung vor dem Zensus. Die Korrekturen sind bei den Männern dabei durchweg größer als bei den Frauen und nehmen mit steigendem Alter (mit Ausnahme der Frauen über Alter 90) leicht ab.

Durch eine Zusammenfassung des Sterblichkeits- und des Zensuseffekts lässt sich der Gesamteffekt - die Veränderung der altersspezifischen Lebenserwartung zwischen der Sterbetafel 2009/11 und der allgemeinen Sterbetafel 2010/12 - darstellen. Bei den Männern gleichen sich der Sterblichkeits- und der Zensuseffekt bis Alter 70 nahezu aus, sodass beide Sterbetafeln sehr ähnliche Werte für die fernere Lebenserwartung ausweisen. Oberhalb von Alter 70 kann der Rückgang der Sterblichkeit den Zensuseffekt nicht mehr kompensieren, sodass die fernere Lebenserwartung in diesem Altersbereich aufgrund des dominierenden Zensuseffekts in der allgemeinen Sterbetafel 2010/12 geringer ist als in der Sterbetafel 2009/11. Bei den Frauen sorgen die geringeren Zensuskorrekturen dafür, dass die fernere Lebenserwartung bis Alter 87 auch auf Basis der Zensusdaten im Zeitraum 2010/12 höher ist als in der Sterbetafel 2009/11. Erst oberhalb von diesem Alter dominiert auch bei den Frauen der Zensuseffekt den Sterblichkeitseffekt, sodass die fernere Lebenserwartung in der Sterbetafel auf Basis der Zensusergebnisse geringer ist als bei der vorangegangen Sterbetafel 2009/11.

Bei der Betrachtung dieser Effekte muss berücksichtigt werden, dass es sich bei der ferneren Lebenserwartung um eine kumulative Maßzahl für die Sterblichkeitsverhältnisse oberhalb des betreffenden Alters handelt. Die Effekte in der absoluten Betrachtung sind unter anderem deswegen in jungen Altersjahren am größten, weil alle Einflüsse der darüber liegenden Altersstufen zusammengefasst werden. Auch wenn relativ gesehen große Korrekturen der Bevölkerungsbestände oberhalb von Alter 90 durch den Zensus stattgefunden haben, sind diese in ihrer absoluten Auswirkung auf die Lebenserwartung marginal. Zum einen ist die fernere Lebenserwartung in diesem Altersbereich bereits sehr gering, zum anderen hat das eingesetzte Extrapolationsverfahren bei der Berechnung von Sterbetafeln auf Fortschreibungsbasis einen Großteil des Fortschreibungsfehlers in den Bevölkerungsdaten im Hinblick auf die Berechnung der Sterbewahrscheinlichkeiten in diesem Altersbereich bereits ausgeglichen. 


\subsection{Auswirkungen des Zensus auf die Messung der Sterblichkeit nach Staatsangehörigkeit}

\subsubsection{Sterblichkeit der deutschen Bevölkerung}

Die Zensuskorrekturen der Bevölkerungszahlen waren für die deutsche Bevölkerung im Vergleich zur gesamten Bevölkerung unterdurchschnittlich - die Bestände der deutschen Männer wurden insgesamt um 1,0\% (-374.398) nach unten korrigiert, die der deutschen Frauen sogar nur um 0,2 \% (-74.085) (Kaus/Mundil-Schwarz 2015: 30). Die stärksten Korrekturen gab es dabei im hohen Alter - die Anzahl der deutschen Männer im Alter von 95 Jahren oder älter wurde durch den Zensus um $64,2 \%(-31.603)$ korrigiert, die der deutschen Frauen in diesem Altersbereich um $17,4 \%(-19.689){ }^{2}$

In Abbildung 2 sind die altersspezifischen Sterbewahrscheinlichkeiten abgebildet, die zum einen mit den Bevölkerungsbeständen der alten Fortschreibung berechnet wurden und zum anderen mit den Daten des Zensus 2011. Betrachtet man die Verläufe der Sterbewahrscheinlichkeiten auf Basis der alten Fortschreibung wird deutlich, dass die Verläufe nur bis etwa Alter 90 plausibel sind. Oberhalb von Alter 90 überschneiden sich die Sterbewahrscheinlichkeiten von Männern und Frauen (mortality crossover) bzw. die Kurven beginnen unplausibel zu sinken. In einer solchen Situation ist es üblich, ein Extrapolationsverfahren einzusetzen, um plausible Verläufe zu generieren (Eisenmenger 2003; Statistisches Bundesamt 2015a). Die extrapolierten Werte sind in Abbildung 2 als gestrichelte Linie dargestellt, die nicht genutzten rohen Beobachtungswerte der Sterbewahrscheinlichkeiten als unverbundene Datenpunkte. Eine Auswirkung auf die gemessene Lebenserwartung bei Geburt hat die Verwendung der extrapolierten Werte dabei nicht: Bei deutschen Männern beträgt der Wert für e(0) mit und ohne Extrapolation 77,7 Jahre, bei Frauen 82,7 Jahre (siehe Tab. 1).

Werden die Sterbetafeln für die deutsche Bevölkerung auf Basis des Zensus 2011 berechnet, dann ist die Lebenswartung bei Geburt für Männer um 0,1 Jahre geringer als auf Basis der alten Fortschreibung (neuer Wert: 77,6 Jahre), bei den Frauen bleibt trotz der geringen Korrekturen der Bevölkerungsbestände ein gemessener Wert von 82,7 Jahren auch auf Basis des Zensus bestehen. Auffällig ist, dass die zensusbasierten Sterbewahrscheinlichkeiten nun bis Alter 100 plausible Werte ergeben, sodass bis zu diesem Alter der Einsatz eines Extrapolationsverfahrens nicht mehr notwendig ist. ${ }^{3}$

2 Berechnung auf Grundlage der Bevölkerungsfortschreibung zum 31.12.2011 auf Basis früherer Zählungen und auf Basis des Zensus 2011.

3 Zur Berechnung einer vollständigen Sterbetafel bis zum Alter 112 wurde wie bei der Vorgehensweise zur allgemeinen Sterbetafel 2010/12 über Alter 100 dennoch ein Extrapolationsverfahren eingesetzt. Sterbewahrscheinlichkeiten oberhalb von Alter 100 beeinflussen die Werte für die Lebenserwartung dabei nur marginal. 
Abb. 2: Altersspezifische Sterbewahrscheinlichkeiten 2010/12 der deutschen Bevölkerung auf Basis der alten Fortschreibung (inkl. Extrapolation) und auf Basis des Zensus 2011

Alte Fortschreibung

log. Sterbewahrscheinlichkeiten

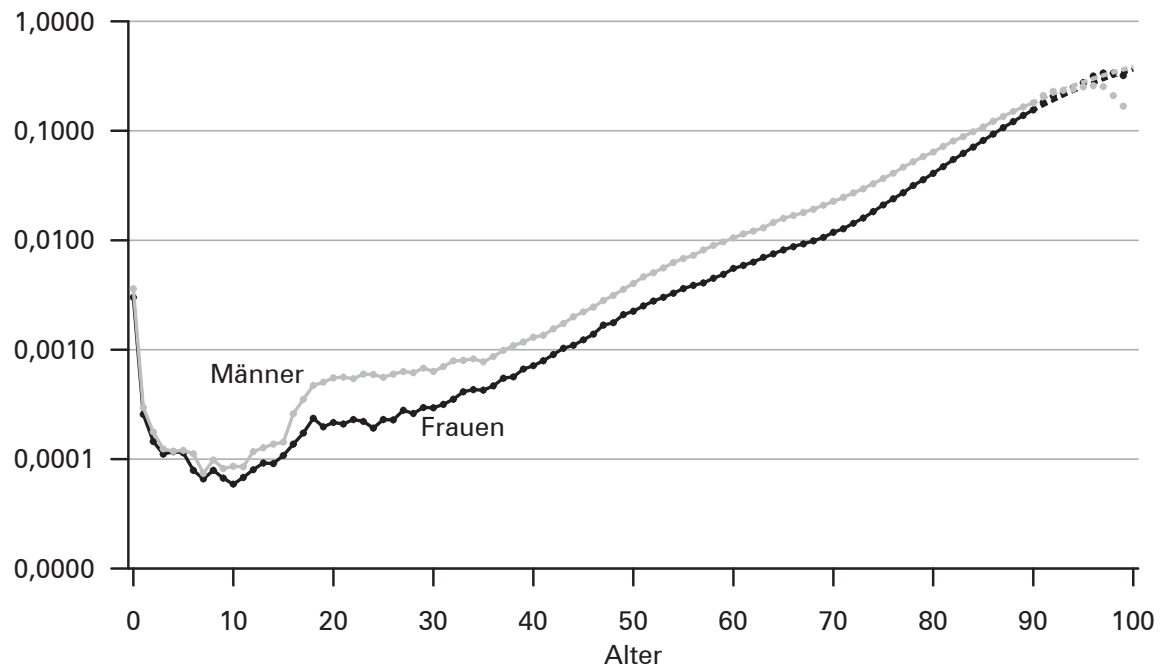

Zensus

log. Sterbewahrscheinlichkeiten

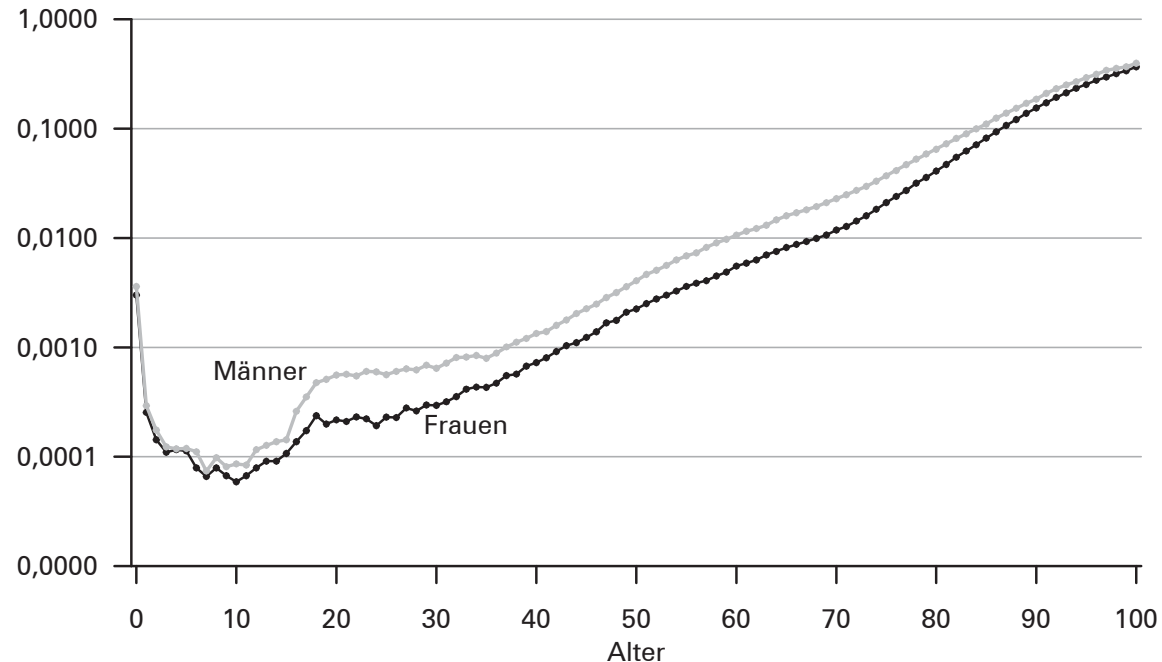

Quelle: Statistisches Bundesamt, eigene Darstellung und Berechnung 
Tab. 1: Lebenserwartung bei Geburt der deutschen Bevölkerung 2010/12 auf Basis verschiedener Berechnungsgrundlagen

\begin{tabular}{lcc}
\hline & Männer & Frauen \\
\hline Alte Fortschreibung & 77,7 & 82,7 \\
Alte Fortschreibung inklusive Extrapolation & 77,7 & 82,7 \\
Zensus & 77,6 & 82,7 \\
Zensus inklusive Extrapolation und Glättung & 77,6 & 82,7 \\
\hline
\end{tabular}

Quelle: Statistisches Bundesamt, eigene Berechnung

In einem nächsten Schritt wurden die zensusbasierten Sterbewahrscheinlichkeiten für die deutsche Bevölkerung geglättet, um einen von Zufallseffekten bereinigten Mortalitätsverlauf über das Alter zu generieren (siehe Abb. 3). Aufgrund der hohen Fallzahlen bei der deutschen Bevölkerung ist der Einfluss des Glättungsverfahrens dabei sehr gering. Der auffälligste Unterschied besteht darin, dass sich die Sterbewahrscheinlichkeiten von Männern und Frauen nun nicht mehr überschneiden, wie es im rohen Verlauf im Alter 4 und 5 der Fall ist. Der Einfluss des Glättungsverfahrens ist auf der logarithmischen Skala etwa bis Alter 35 sichtbar, oberhalb von diesem Alter sind rohe und ausgeglichene Werte praktisch identisch.

Abb. 3: Altersspezifische Sterbewahrscheinlichkeiten 2010/12 der deutschen Bevölkerung auf Basis des Zensus 2011: rohe und ausgeglichene Werte log. Sterbewahrscheinlichkeiten

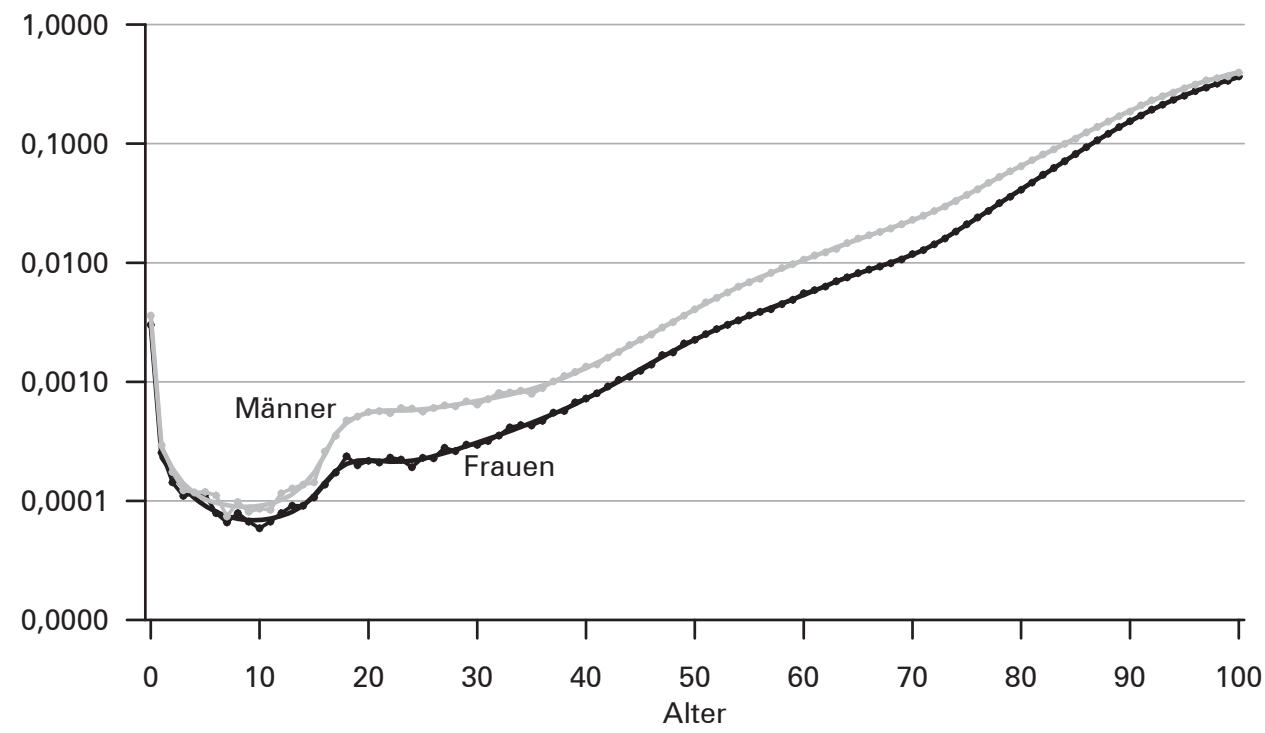

Quelle: Statistisches Bundesamt, eigene Darstellung und Berechnung 
Abb. 4: Differenzen in der altersspezifischen Lebenserwartung zwischen einer Sterbetafel 2010/12 für die deutsche Bevölkerung auf Fortschreibungsund auf Zensusbasis (Zensuseffekt)

e(x)-Differenzen in Jahren

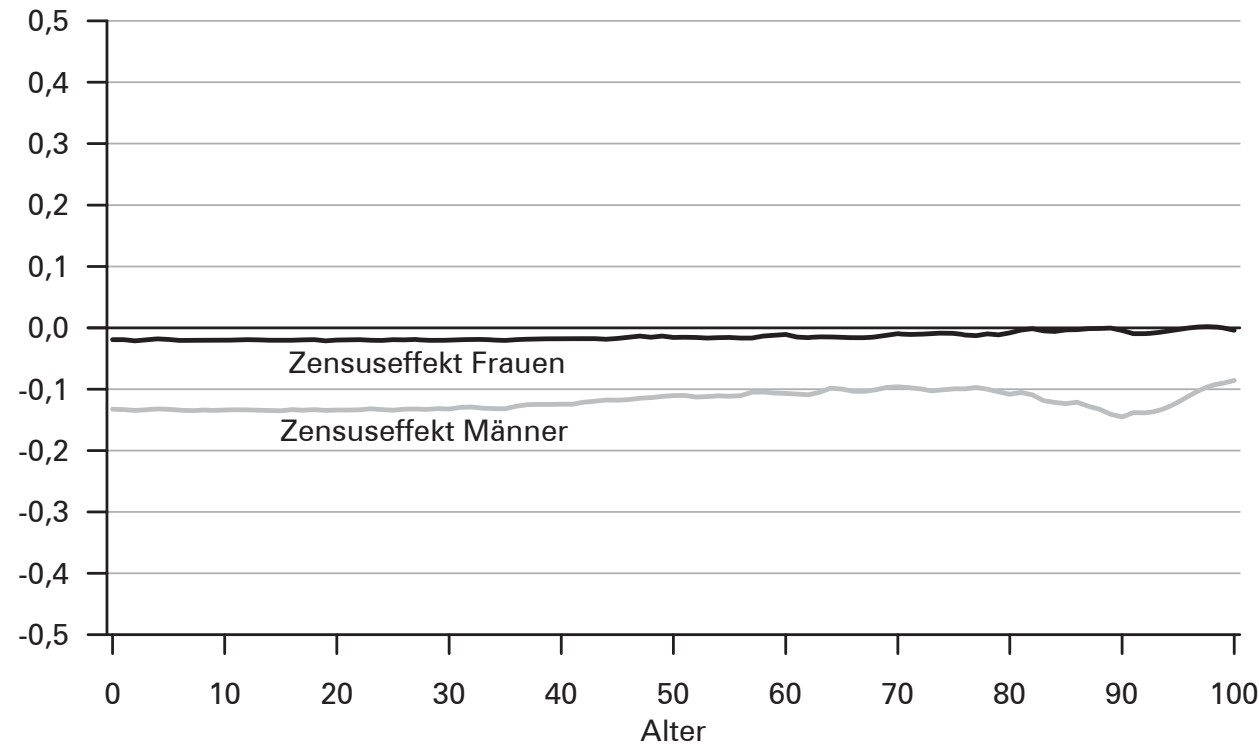

Quelle: Statistisches Bundesamt, eigene Darstellung und Berechnung

Der Verlauf der ausgeglichenen Sterbewahrscheinlichkeiten für die deutsche Bevölkerung und die auf diesen Werten aufbauende Sterbetafel wird im Folgenden genutzt, um die grundlegenden Sterblichkeitsmuster der deutschen Bevölkerung zu analysieren und mit den entsprechenden Ergebnissen der ausländischen Bevölkerung zu vergleichen. Betrachtet man beispielsweise den Verlauf der altersspezifischen (ferneren) Lebenserwartung auf Basis dieser Werte im Vergleich zu Werten auf Basis der alten Fortschreibung, wird noch einmal besonders deutlich, wie gering die Korrekturen durch den Zensus im Hinblick auf die Sterblichkeitsmessung für die deutsche Bevölkerung sind. Die fernere Lebenserwartung der deutschen Frauen wird nur marginal beeinflusst, sodass selbst die erste Nachkommastelle in jedem Altersjahr auf Basis des Zensus und auf Basis der alten Fortschreibung identisch ist. Bei den Männern wird die fernere Lebenserwartung über den gesamten Altersverlauf hinweg auf Zensusbasis um 0,1 Jahre nach unten korrigiert.

\subsubsection{Sterblichkeit der ausländischen Bevölkerung}

Die Zensuskorrekturen der ausländischen Bevölkerung waren besonders ausgeprägt: Die Bestände der Männer wurden insgesamt um 15,9 \% (-602.318) nach unten korrigiert, die der Frauen um 12,9 \% (-465.042) (Kaus/Mundil-Schwarz 2015: 30). Auch bei der ausländischen Bevölkerung gab es die stärksten Korrekturen im hohen 
Alter. Bereits oberhalb von Alter 75 wurden die Bestände um 36,2 \% (-46.008 Männer) bzw. 38,9 \% (-51.242 Frauen) durch den Zensus reduziert. Oberhalb von Alter 95 betrugen die Korrekturen sogar 94,5 \% bei den Männern und 90,6 \% bei den Frauen. Die zensuskorrigierte Bevölkerungsfortschreibung hat in dieser Altersgruppe zum 31.12.2011 statt 23.561 Personen auf alter Basis nur 1.831 Personen ermittelt (547 Männer und 1.284 Frauen).

Abbildung 5 zeigt die auf Basis der alten Fortschreibung und auf Basis des Zensus 2011 ermittelten altersspezifischen Sterbewahrscheinlichkeiten der ausländischen Bevölkerung. Auffällig ist, dass auf Grundlage der alten Fortschreibung bereits ab Alter 75 eine nicht plausible Entwicklung im Verlauf der altersspezifischen Sterbewahrscheinlichkeiten auftritt: Die Sterbewahrscheinlichkeiten der Männer steigen auf einem niedrigeren Niveau als es die vorangegangen Altersstufen suggerieren würden, sodass es bereits im Alter 84 zu einer Überschneidung der männlichen und weiblichen Sterbewahrscheinlichkeiten kommt. Durch Einsatz des Extrapolationsverfahrens (gestrichelte Linie) lässt sich jedoch eine plausible Fortsetzung des Verlaufs oberhalb von Alter 75 generieren. Da bei diesem Vorgehen im Vergleich zur deutschen Bevölkerung viel früher im Altersverlauf rohe Werte durch (höhere) Werte aus dem Extrapolationsmodell ersetzt werden, ist der Einfluss auf die gemessene (fernere) Lebenserwartung entsprechend größer. Auf Basis der rohen Werte würde man eine Lebenserwartung bei Geburt der ausländischen Männer von 84,8 Jahren ermitteln, für Frauen von 88,3 Jahren. Durch den Einsatz des Extrapolationsverfahrens reduzieren sich die Werte auf 82,9 Jahre bei männlichen (-1,9 Jahre) und 87,8 Jahre bei weiblichen Neugeborenen (-0,5 Jahre) erheblich.

Auf Basis des Zensus 2011 lassen sich plausiblere Sterbewahrscheinlichkeiten bis in deutlich höhere Altersstufen ableiten als auf Grundlage der alten Fortschreibung. Die zensuskorrigierte Lebenserwartung der ausländischen Männer, berechnet mit den rohen Daten, beträgt 80,9 Jahre, die der Frauen 85,0 Jahre. Trotz der größeren Korrekturen bei den Bevölkerungszahlen der Männer ist die Zensuskorrektur bei den Frauen (-2,8 Jahre) größer als bei den Männern (-2,0 Jahre). Dies ist lediglich darauf zurückzuführen, dass die Extrapolation auf Basis der Fortschreibung bei den Männern deutlich stärker gewirkt hat als bei den Frauen. Ein Vergleich dieser Werte ist trotzdem sinnvoll, da ein Einsatz des Extrapolationsverfahrens auf Basis der alten Fortschreibung zwingend notwendig gewesen wäre, um eine in sich plausible Sterbetafel für die ausländische Bevölkerung aufzustellen.

Um ein charakteristisches Mortalitätsmuster für die ausländische Bevölkerung abzuleiten, ist auch auf Zensusbasis nicht nur der Einsatz eines Glättungsverfahrens, sondern auch der eines Extrapolationsverfahrens vor Alter 100 notwendig. In Abbildung 6 ist dieser Verlauf dargestellt und den rohen Beobachtungswerten gegenübergestellt. Die ungenutzten rohen Beobachtungswerte sind dabei wie in den vorangegangenen Abbildungen als einzelne Datenpunkte dargestellt, während die in das Glättungsverfahren eingeflossenen Werte miteinander verbunden sind. Der geglättete Verlauf ist als durchgezogene Linie dargestellt, die extrapolierten Werte als gestrichelte Line. Auf Basis des Zensus wurden die geglätteten Verläufe der Männer ab Alter 91, die der Frauen ab Alter 92 durch extrapolierte Werte ergänzt. Die auf Grundlage dieser methodischen Eingriffe ermittelten Sterbewahrscheinlich- 
Abb. 5: Altersspezifische Sterbewahrscheinlichkeiten 2010/12 der nichtdeutschen Bevölkerung auf Basis der alten Fortschreibung (inkl. Extrapolation) und auf Basis des Zensus 2011

Alte Fortschreibung

log. Sterbewahrscheinlichkeiten

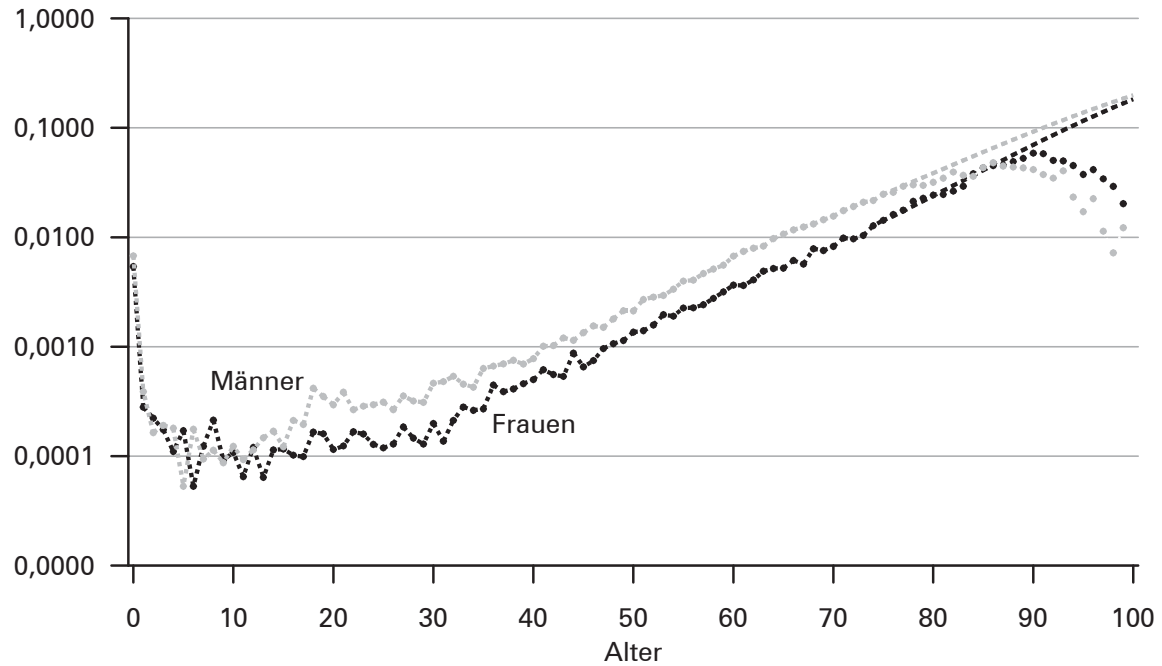

Zensus

log. Sterbewahrscheinlichkeiten

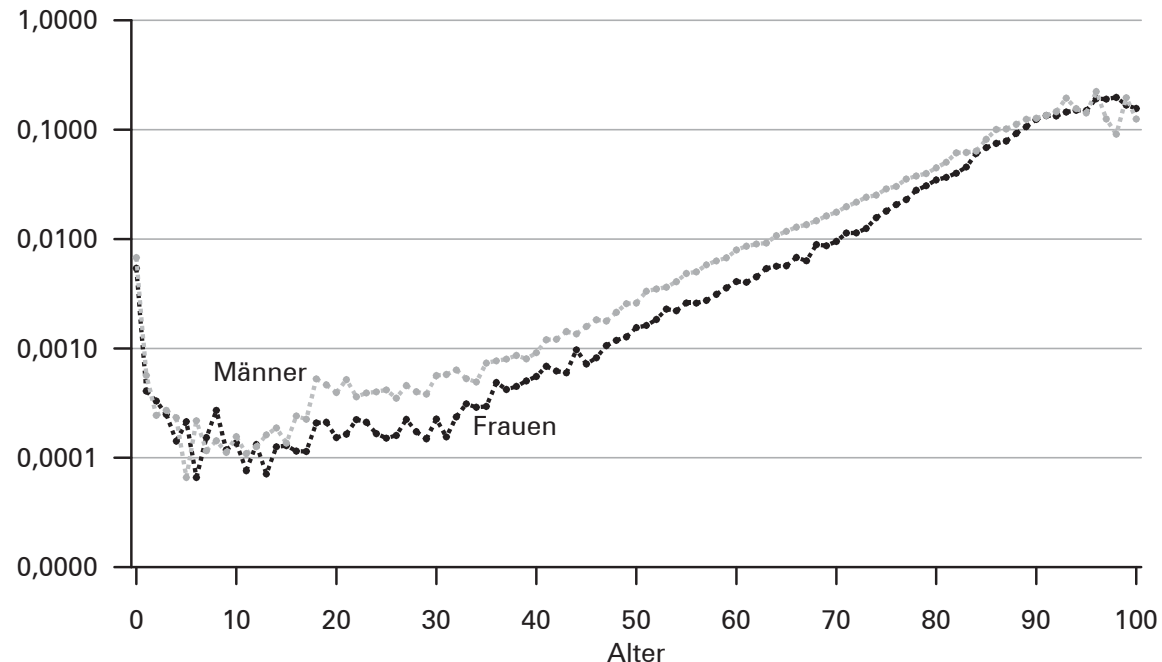

Quelle: Statistisches Bundesamt, eigene Darstellung und Berechnung

keiten resultieren in einer Lebenserwartung bei Geburt von 80,5 Jahren bei den ausländischen Männern und 84,8 Jahren bei den Frauen und sind somit noch einmal etwas geringer als die auf Basis der rohen Daten ermittelten Werte (siehe Tab. 2). 
Abb. 6: Altersspezifische Sterbewahrscheinlichkeiten 2010/12 der nichtdeutschen Bevölkerung auf Basis des Zensus 2011: rohe und ausgeglichene Werte

log. Sterbewahrscheinlichkeiten

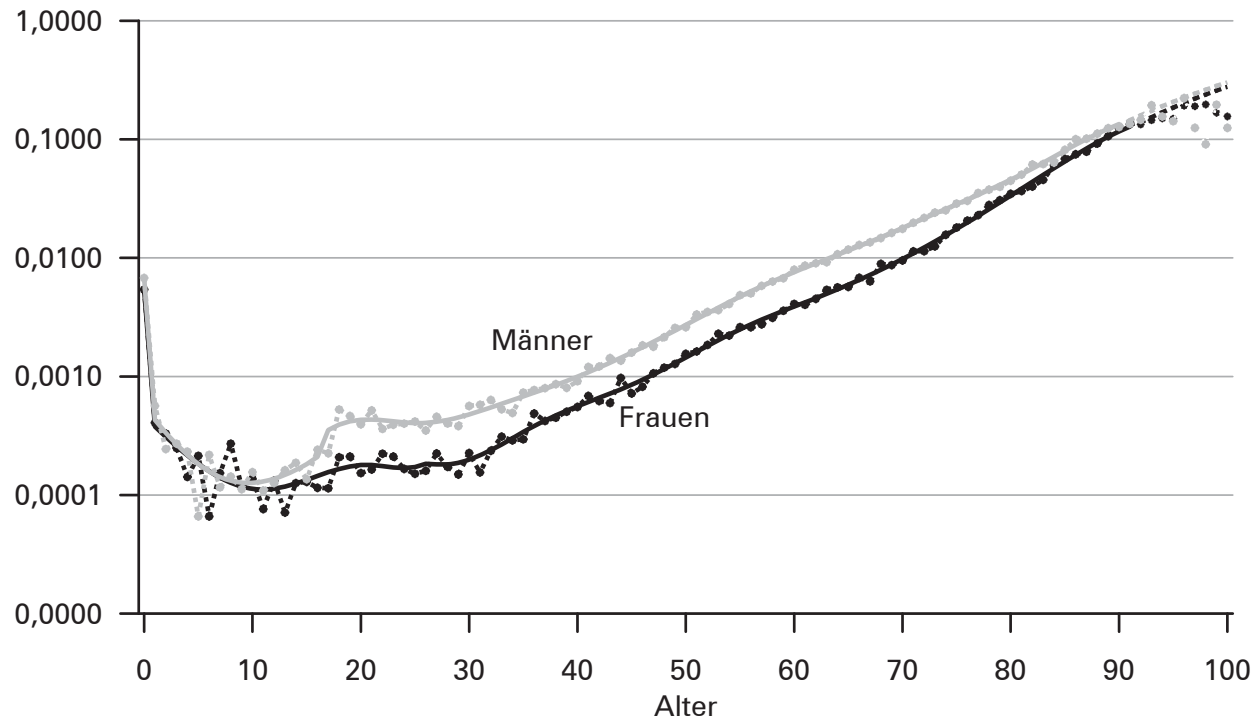

Quelle: Statistisches Bundesamt, eigene Darstellung und Berechnung

Tab. 2: Lebenserwartung bei Geburt der nichtdeutschen Bevölkerung 2010/12 auf Basis verschiedener Berechnungsgrundlagen

\begin{tabular}{lcc}
\hline & Männer & Frauen \\
\hline Alte Fortschreibung & 84,8 & 88,3 \\
Alte Fortschreibung inklusive Extrapolation & 82,9 & 87,8 \\
Zensus & 80,9 & 85,0 \\
Zensus inklusive Extrapolation und Glättung & 80,5 & 84,8 \\
\hline
\end{tabular}

Quelle: Statistisches Bundesamt, eigene Berechnung

Zieht man die in Abbildung 6 dargestellten geglätteten Verläufe der altersspezifischen Sterbewahrscheinlichkeiten zur Berechnung einer kompletten Sterbetafel heran und vergleicht diese Werte mit denen der alten Bevölkerungsfortschreibung, lässt sich ein Zensuseffekt von 2,4 Jahren bei den Männern und von 3,0 Jahren bei den Frauen für die Lebenserwartung bei Geburt konstatieren. Abbildung 7 zeigt den Verlauf des Zensuseffekts für die (fernere) Lebenserwartung im gesamten Altersbereich. Bei den Männern reduzieren sich die Werte für die e(x)-Differenz bis Alter 85 infolge des Zensus nur leicht - in diesem Alter beträgt der Zensuseffekt immer noch 2 Jahre, bevor der Einfluss des Zensus deutlich abnimmt. Selbst die fernere Lebenserwartung im Alter 100 ist trotz des Einsatzes eines Extrapolationsverfah- 
Abb. 7: Differenzen in der altersspezifischen Lebenserwartung zwischen einer Sterbetafel 2010/12 für die nichtdeutsche Bevölkerung auf Fortschreibungs- und auf Zensusbasis (Zensuseffekt)

$\mathrm{e}(\mathrm{x})$-Differenzen in Jahren

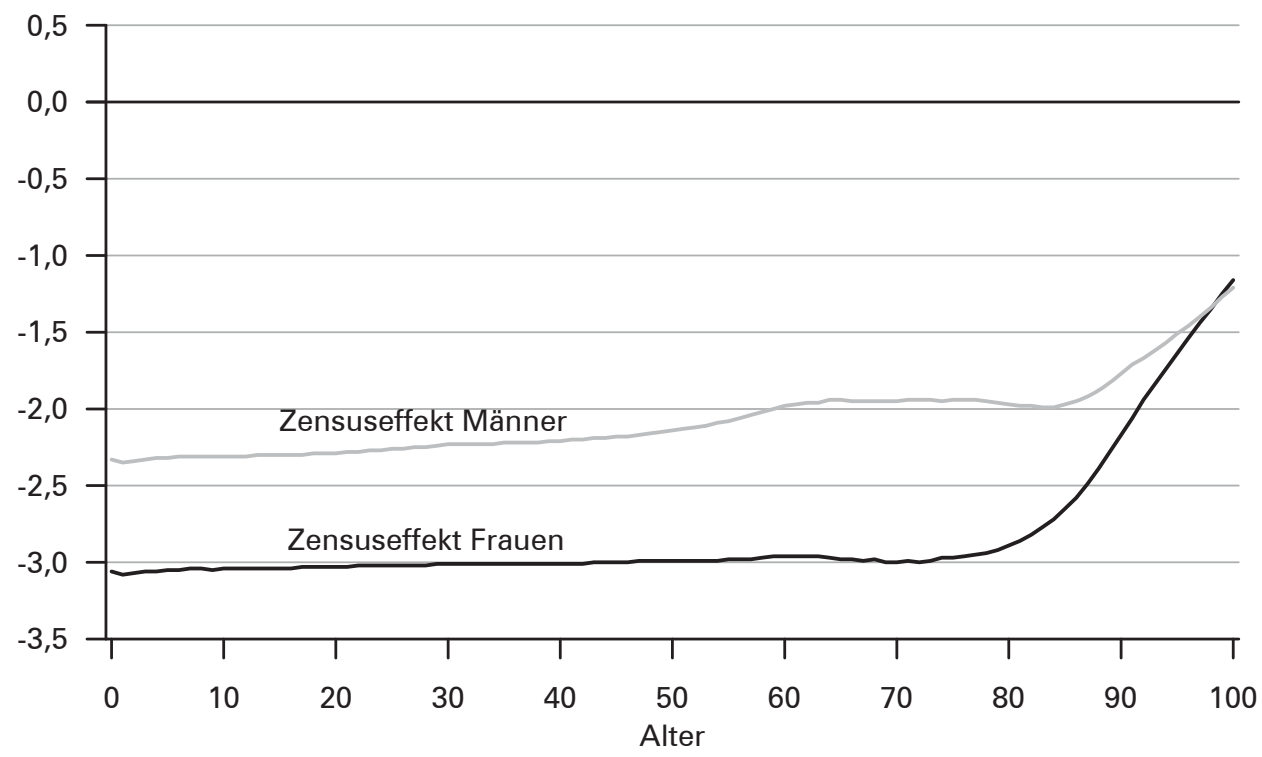

Quelle: Statistisches Bundesamt, eigene Darstellung und Berechnung

rens bei den Daten der alten Fortschreibung noch um 1,2 Jahre reduziert, wenn die Daten des Zensus der Berechnung zugrunde gelegt werden. Bei den Frauen bleibt der Einfluss des Zensus von 3 Jahren auf die fernere Lebenserwartung bis über das Alter 70 hinaus nahezu konstant, bevor er ebenfalls bis auf einen Wert von 1,2 Jahren im Alter 100 abnimmt.

\subsubsection{Vergleich der Sterblichkeit von Deutschen und Nichtdeutschen}

In den vorangegangen Abschnitten wurden durch methodische Eingriffe wie die Glättung und den Einsatz eines Extrapolationsverfahrens charakteristische Verlaufsmuster der altersspezifischen Sterbewahrscheinlichkeiten für Deutsche und Ausländer auf Basis der Daten des Zensus 2011 vorgestellt. In Abbildung 8 sind diese Verläufe getrennt nach Geschlecht abgebildet. Bei den durchgezogenen Linien handelt es sich um die geglätteten Verläufe. Werte, die bei der ausländischen Bevölkerung mithilfe des Extrapolationsverfahrens modelliert wurden, sind gestrichelt dargestellt.

Bei Betrachtung der jeweiligen Verläufe wird deutlich, dass sich die Sterblichkeit zwischen deutscher und nichtdeutscher Bevölkerung bei Männern und Frauen auf sehr ähnliche Art und Weise unterscheidet. Bei Säuglingen, im Kindesalter sowie im frühen Jugendalter weisen Personen mit ausländischer Staatsangehörigkeit eine 
Abb. 8: Altersspezifische Sterbewahrscheinlichkeiten 2010/12 für deutsche und nichtdeutsche Bevölkerung

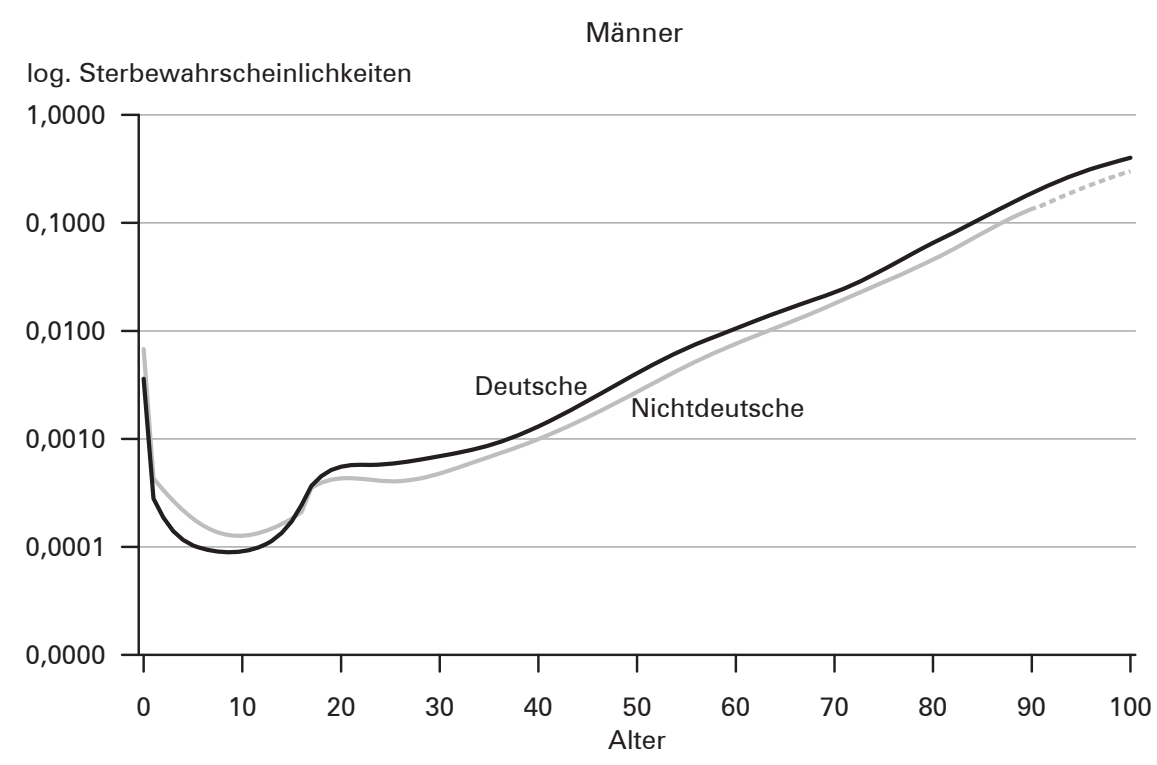

Frauen

log. Sterbewahrscheinlichkeiten

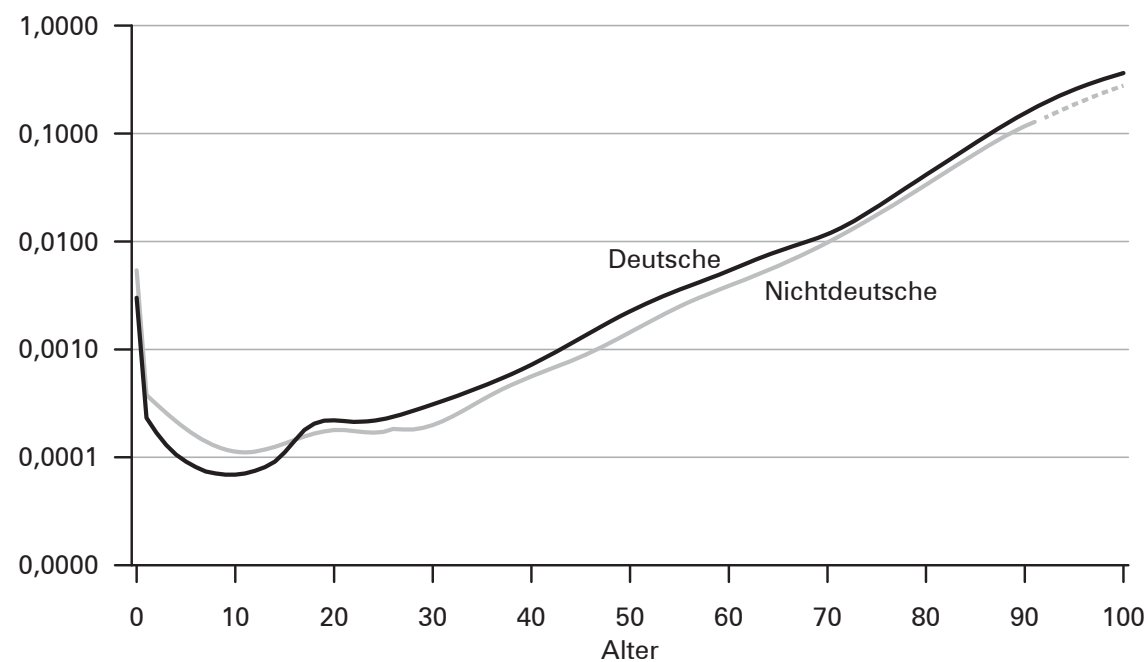

Quelle: Statistisches Bundesamt, eigene Darstellung und Berechnung

höhere Sterblichkeit auf als gleichaltrige Personen mit deutscher Staatsbürgerschaft. Bei den Jungen kehrt sich das Verhältnis im Alter 16 um, bei den Mädchen im Alter 17. In allen folgenden Altersjahren weisen ausländische Männer und Frauen stets geringere Sterbewahrscheinlichkeiten auf als gleichaltrige Deutsche. Berech- 
net man Konfidenzintervalle für die Verläufe der Sterbewahrscheinlichkeiten, lässt sich nachweisen, dass die Unterschiede bei den neugeborenen Jungen und Mädchen signifikant sind, bei den darauffolgenden Altersjahren im Kindes- und Jugendalter jedoch nicht. Durch die geringen Fallzahlen bei der ausländischen Bevölkerung sind die Konfidenzintervalle in diesem Altersbereich entsprechend breit, sodass mit einer $5 \%$ igen Irrtumswahrscheinlichkeit nicht auf signifikante Unterschiede geschlossen werden kann.

Signifikante Unterschiede in den Sterbewahrscheinlichkeiten von Deutschen und Nichtdeutschen finden sich jedoch bei Männern in den Altersjahren 25 bis 32 und oberhalb von Alter 38. Bei den Frauen sind die Unterschiede im Alter 31 signifikant und in allen Altersjahren oberhalb von Alter 41. Für die extrapolierten Werte lassen sich keine Konfidenzintervalle in gleicher Weise berechnen, sodass oberhalb von Alter 91 bei den Männern bzw. Alter 92 bei den Frauen keine vergleichbaren Aussagen über die Signifikanz der Unterschiede getroffen werden können. Die extrapolierten Werte für die ausländische Bevölkerung liegen bei Männern und Frauen jedoch außerhalb des für die Sterbewahrscheinlichkeiten der deutschen Bevölkerung berechneten Konfidenzintervalls, was auf signifikante Unterschiede hindeutet.

In Abbildung 9 sind die Sterblichkeitsunterschiede zwischen Deutschen und Nichtdeutschen noch einmal prozentual dargestellt (Maßzahl $U_{x}$ ). Aus der Darstellung lässt sich beispielsweise ablesen, dass die Säuglingssterblichkeit der mit ausländischer Staatsbürgerschaft geborenen Kinder um etwa 80 bis $90 \%$ höher ist als die der deutschen Kinder. In den darauffolgenden Altersjahren sind die Unterschiede selbst bei einem dreijährigen Betrachtungszeitraum aufgrund der geringen Fallzahlen bei den Sterbefällen nicht signifikant und sollten entsprechend nicht überinterpretiert werden.

Grundlegend passen die Verläufe jedoch zu früheren Befunden nach denen ausländische Kinder bis ins Jugendalter hinein eine erhöhte Sterblichkeit im Vergleich zur deutschen Bevölkerung haben. Oberhalb von Alter 25 sind die Unterschiede jedoch weitestgehend signifikant und schwanken zwischen 20 und $35 \%$ niedrigeren Sterbewahrscheinlichkeiten der Ausländerinnen und Ausländer im Vergleich zu den Deutschen.

Ein klares Muster, nach dem die Unterschiede im Erwerbsalter besonders stark sind und mit höherem Alter abnehmen bzw. sich sogar in eine höhere Sterblichkeit der ausländischen Bevölkerung umwandeln, wie es frühere Untersuchungen nahegelegt haben, lässt sich auf Basis der Daten des Zensus nicht ableiten. Bei Männern ist eine solche Tendenz nicht zu erkennen, bei den Frauen nur ansatzweise, da die Sterblichkeitsunterschiede zwischen Alter 65 und 72 deutlich zurückgehen, in den darauffolgenden Altersjahren jedoch wieder zunehmen.

Der Unterschied in der Lebenserwartung bei Geburt zwischen Deutschen und Nichtdeutschen beläuft sich bei den zensusbasierten und von Zufallseinflüssen bereinigten Daten auf 2,9 Jahre bei den Männern und 2,1 Jahre bei den Frauen. Im Alter 17, dem Alter, ab dem die ausländische Bevölkerung bei Männern und Frauen durchweg niedrigere Sterbewahrscheinlichkeiten aufweist, betragen die Unterschiede in der ferneren Lebenserwartung sogar 3,3 Jahre bei den Männern und 2,3 Jahre bei den Frauen. Auch im Alter 65 sind noch erhebliche Unterschiede festzustellen - die 
Abb. 9: Altersspezifische Unterschiede zwischen den

Sterbewahrscheinlichkeiten der nichtdeutschen und der deutschen Bevölkerung nach Geschlecht auf Basis des Zensus für den Zeitraum 2010/12

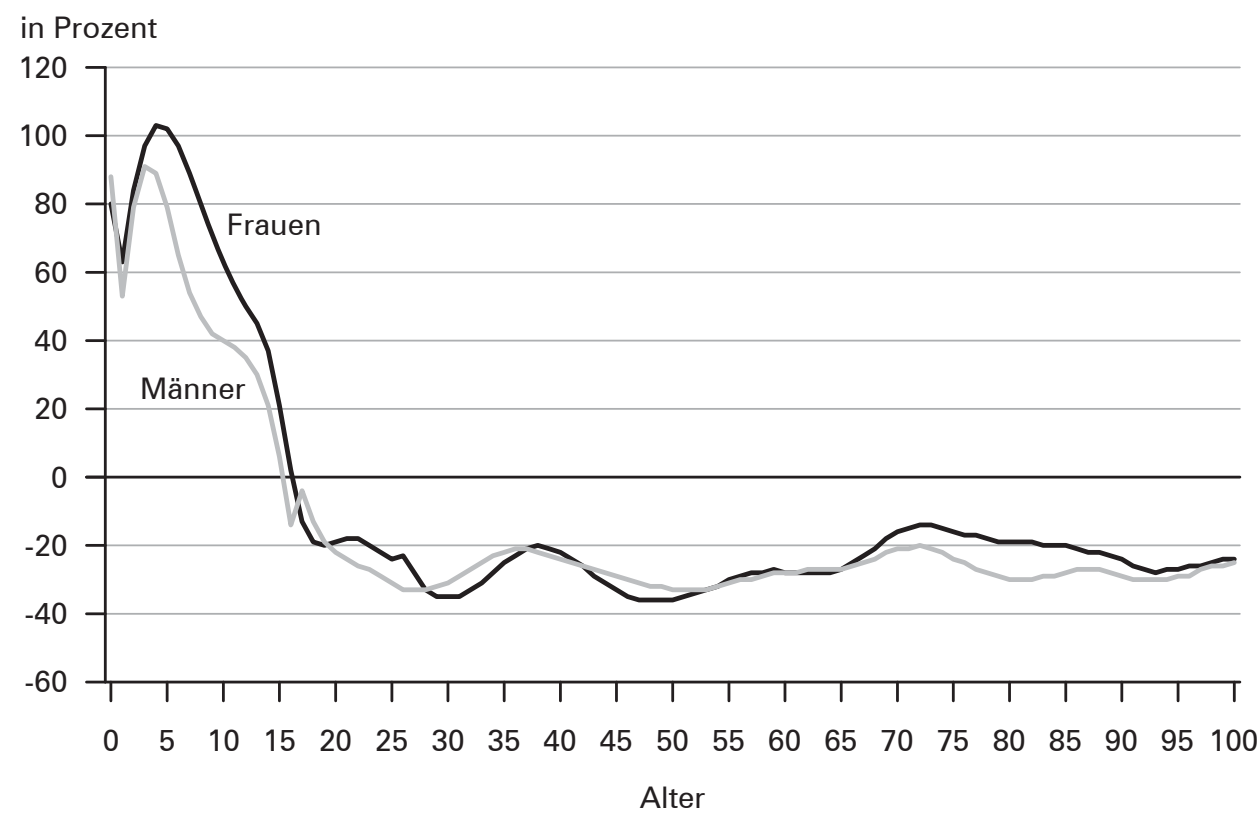

Quelle: Statistisches Bundesamt, eigene Darstellung und Berechnung

fernere Lebenserwartung der ausländischen Männer ist um 2,4 Jahre höher, die der Frauen um 1,7 Jahre im Vergleich zu gleichaltrigen deutschen Personen (siehe auch Tab. 3).

Tab. 3: Fernere Lebenserwartung in ausgewählten Altersstufen für deutsche und nichtdeutsche Bevölkerung 2010/12 (auf Basis des Zensus 2011 - Sterbetafeln mit ausgeglichenen Sterbewahrscheinlichkeiten und extrapolierten Werten in hohem Alter)

\begin{tabular}{lcccc}
\hline \multirow{2}{*}{$\begin{array}{l}\text { Ferne Lebenserwartung } \\
\text { im Alter von ... }\end{array}$} & \multicolumn{2}{c}{ Männer } & \multicolumn{2}{c}{ Frauen } \\
\hline 0 Jahren & 77,6 & 80,5 & 82,7 & 84,8 \\
20 Jahren & 58,1 & 61,4 & 63,1 & 65,5 \\
40 Jahren & 38,8 & 41,9 & 43,5 & 45,8 \\
65 Jahren & 17,4 & 19,8 & 20,7 & 22,4 \\
80 Jahren & 7,7 & 9,5 & 9,2 & 10,6 \\
\hline
\end{tabular}

Quelle: Statistisches Bundesamt, eigene Berechnung 


\section{Diskussion}

Die Daten des Zensus 2011 haben es ermöglicht, grundlegende Sterblichkeitsmuster in Deutschland zu analysieren. Der große Vorteil der Zensusdaten liegt darin, dass die Bevölkerungsbestände möglichst genau erhoben wurden und diese frei von einem Fortschreibungs- bzw. einem Rückrechnungsfehler sind. Ein Fortschreibungsfehler in den Bevölkerungsdaten galt als Hauptursache dafür, dass insbesondere die Lebenserwartung der ausländischen Bevölkerung als überhöht angegeben wurde. Die Ergebnisse der allgemeinen Sterbetafel 2010/12 haben bereits für die gesamte Bevölkerung Deutschlands gezeigt, dass die Lebenserwartung auf Basis des Zensus etwas geringer ist auf Basis der alten Fortschreibung. Bei den Männern entsprach der Wert für die Lebenserwartung bei Geburt auf Grundlage des Zensus genau dem Wert, den auch die letzte Sterbetafel auf Basis der alten Fortschreibung für den Zeitraum 2009/11 hervorgebracht hat. Die vorliegenden Analysen haben gezeigt, dass der Zensuseffekt auf die Lebenserwartung neugeborener Jungen dabei genau so groß war wie der Rückgang der Sterblichkeit zwischen beiden Betrachtungszeiträumen und sich die Werte deswegen genau entsprechen. Durch die geringeren zensusbedingten Korrekturen der Bevölkerungsbestände bei den Frauen bei einem ähnlich großen Rückgang der Sterblichkeit zwischen 2009/11 und 2010/12 ist die Lebenserwartung neugeborener Mädchen im Vergleich beider Sterbetafeln leicht angestiegen (+ 0,1 Jahre). Erst im hohen Alter ist die fernere Lebenserwartung auf Zensusbasis bei beiden Geschlechtern infolge der großen (relativen) Bevölkerungskorrekturen in diesem Altersbereich etwas geringer als in der letzten Sterbetafel, die auf Basis der alten Fortschreibung berechnet worden ist (2009/11). Auf Ebene der Gesamtbevölkerung waren die Auswirkungen des Zensus folglich relativ gering und bieten keinen Anhaltspunkt für eine generelle Neubewertung der Sterblichkeit in Deutschland. Die Werte wurden in etwa um das Ausmaß der derzeit beobachtbaren Sterblichkeitsveränderungen zwischen zwei Berechnungszeiträumen korrigiert.

Deutlichere Auswirkungen auf die Höhe der gemessenen Lebenserwartung durch den Zensus zeigen sich bei einer differenzierten und den thematisierten methodischen Einschränkungen unterworfenen Betrachtung nach Staatsangehörigkeit. Die Lebenswartung der deutschen Bevölkerung wurde bei den Frauen praktisch überhaupt nicht beeinflusst, die der Männer ist um 0,1 Jahre geringer als auf Basis der alten Fortschreibung. Entsprechend größere Korrekturen gab es bei der ausländischen Bevölkerung. Die Lebenserwartung bei Geburt von ausländischen Männern ist um 2,4 Jahre, die ausländischer Frauen um 3,0 Jahre geringer als Werte, die für den gleichen Betrachtungszeitraum auf Basis der alten Fortschreibungsdaten ermittelt worden wären. Bei diesem Vergleich ist der Einfluss eines Extrapolationsverfahrens zu berücksichtigen, dessen Einsatz die Lebenserwartung der ausländischen Bevölkerung bereits auf Basis der alten Fortschreibungsdaten deutlich nach unten korrigiert hat - bei den Männern stärker als bei den Frauen. Die zuvor extrapolierten Werte im hohen Alter haben die auf Grundlage des Zensus zuverlässiger ermittelbaren Sterbewahrscheinlichkeiten dennoch unterschätzt. Der Grund hierfür liegt darin, dass die Sterbewahrscheinlichkeiten im Stützbereich der Extrapolation zensusbedingt ebenfalls nach oben korrigiert werden mussten. Auf Basis des Zensus 
lassen sich bis in deutlich höhere Altersjahre plausible Sterbewahrscheinlichkeiten der ausländischen Bevölkerung ermitteln, sodass auch die dennoch notwendige Extrapolation oberhalb von Alter 91 (Männer) bzw. 92 (Frauen) auf zuverlässigere Stützwerte zurückgreifen kann und sich insgesamt plausiblere Mortalitätsmuster ergeben. Diese Ergebnisse zeigen, wie groß der Einfluss des Extrapolationsverfahrens bei der Aufstellung von Sterbetafeln für Teilpopulationen sein kann. Entsprechend elementar ist es, bei vergleichbaren Studien die Hintergrundinformationen zur Extrapolation, wie das Startalter und das verwendeten Verfahren, offenzulegen und zu begründen.

Vergleicht man die charakteristischen Sterblichkeitsmuster der deutschen und der ausländischen Bevölkerung über den gesamten Altersbereich hinweg, zeigt sich kein grundlegend neues Bild auf Basis des Zensus, es werden jedoch deutlich reduzierte Unterschiede im Vergleich zu Analysen auf Basis der alten Fortschreibung sichtbar. Der Vorteil der ausländischen Bevölkerung in der Höhe der Lebenserwartung bei Geburt reduziert sich von 5,2 Jahren auf 2,9 Jahre bei den Männern und von 5,1 Jahren auf 2,1 Jahre bei den Frauen. Auch oberhalb von Alter 65 bleibt ein deutlicher und in weiten Teilen signifikanter Sterblichkeitsvorteil der ausländischen Bevölkerung bestehen. Die relativen Differenzen in den altersspezifischen Sterbewahrscheinlichkeiten unterscheiden sich dabei in hohem Alter kaum von denen im Erwerbsalter. Die Befunde deuten dabei auf einen starken Selektionseffekt (Healthy-Migrant-Effekt) hin. Hinweise auf einen Adaptionseffekt, der für eine deutliche Angleichung der Sterblichkeitsverhältnisse zwischen der deutschen und der ausländischen Bevölkerung in höherem Alter sorgen würde, lassen sich mit den zensusbasierten Ergebnissen nicht finden. Dabei können allerdings zusätzliche Selektionseffekte beispielsweise bei der Einbürgerung oder einer selektiven Abwanderung eine Rolle spielen. Mit zunehmendem Alter steigt auch der Anteil an eingebürgerten Personen an der ursprünglichen ausländischen Bevölkerung an. Allerdings hat es in den letzten Jahrzehnten kein gleichmäßiges Einbürgerungsverhalten gegeben, was die Bewertung dieses Prozesses erschwert. Zudem besteht die Möglichkeit, dass Personen mit einer nichtdeutschen Staatsangehörigkeit und einer erhöhten Morbidität tendenziell eher zurück in das jeweilige Herkunftsland wandern. Deswegen lässt sich auf Basis der vorliegenden Analysen nicht ausschließen, dass es auf der Individualebene durchaus einen Adaptionseffekt gibt, auch wenn er sich auf der Makroebene nicht nachweisen lässt. Eine signifikant höhere Sterbewahrscheinlichkeit von Nichtdeutschen lässt sich nur für die Säuglingssterblichkeit bei beiden Geschlechtern nachweisen. Die Ergebnisse deuten jedoch auch auf eine höhere Sterbewahrscheinlichkeit im Kindes- und frühen Jugendalter unter der ausländischen Bevölkerung hin, auch wenn die Unterschiede in diesem Altersbereich nicht signifikant sind. Insgesamt bestätigen die Ergebnisse damit, dass die ausländische Bevölkerung, trotz gegebener sozio-ökonomischer Benachteiligun- 
gen, ${ }^{4}$ eine deutlich geringere Sterblichkeit als die deutsche Bevölkerung aufweist. Diese Konstellation kann als paradox bezeichnet werden, da sozio-ökonomische Benachteiligungen in zahlreichen Studien mit einer höheren Sterblichkeit in Verbindung gebracht werden. ${ }^{5}$

Die vorgelegten Ergebnisse zeichnen kein völlig neues Bild der Sterblichkeitsverhältnisse in Deutschland. Bei Betrachtung der Gesamtbevölkerung zeigen sich nur geringe Effekte auf die gemessene Lebenserwartung durch die Berücksichtigung der Bevölkerungsbestände des Zensus 2011. Dahinter verbergen sich jedoch interessante Befunde im Hinblick auf die Sterblichkeit der ausländischen Bevölkerung und deren Unterschiede zur Sterblichkeit der Bevölkerung mit deutscher Staatsangehörigkeit. Ein Healthy-Migrant-Effekt und damit eine geringere Sterblichkeit der ausländischen Bevölkerung - wenn auch in deutlich geringerem Ausmaß als auf Basis der alten Fortschreibung - kann auch unter Ausschluss von Fehlern in der Bevölkerungsfortschreibung mithilfe der Daten des Zensus 2011 nachgewiesen werden. Eine überproportionale Untererfassung der ausländischen Bevölkerung in der Bevölkerungsfortschreibung war zuvor als eine der wesentlichen Ursachen für die deutlich höhere Lebenserwartung der ausländischen Bevölkerung auf Basis dieser Daten angeführt worden (Luy 2007; Kibele et al. 2008; Koh/s 2008a/b, 2011, 2012; Mammey/Schwarz 2002; Robert Koch Institut/Statistisches Bundesamt 2008). Um die Bedeutung des Healthy-Migrant-Effektes abzuschätzen, haben Forscher in der Vergangenheit deshalb auf alternative Datenquellen (z.B. das AZR, die Daten der GRV oder den Integrationssurvey) zurückgegriffen. Diese Studien waren jedoch anderen Einschränkungen unterworfen, da sie nicht auf eine Erhebung der gesamten Bevölkerung und der Sterbefälle zurückzugreifen, um Sterblichkeitsunterschiede zwischen der deutschen und der ausländischen Bevölkerung analysieren zu können (siehe Abschnitt 4.1). Die hier vorgelegten Befunde zur Sterblichkeit der deutschen und ausländischen Bevölkerung ermöglichen es nun auf Basis des Zensus mit größtmöglicher Sicherheit auszuschließen, dass es sich bei den bisherigen Befunden zum Healthy-Migrant-Effekt nur um ein Artefakt gehandelt hat. Zukünftige Forschung kann sich unter Berücksichtigung dieses Ergebnisses wieder stärker mit inhaltlichen Erklärungen für die nachgewiesenen Sterblichkeitsunterschiede zwischen der deutschen und der ausländischen Bevölkerung auseinandersetzen. Die im Online-Anhang zur Verfügung gestellten Sterbetafeln für die deutsche und die nicht-deutsche Bevölkerung ermöglichen es, dabei auch auf altersspezifische Unterschiede genauer einzugehen.

4 Für eine ausführliche Beschreibung der sozio-ökonomischen Situation der ausländischen Bevölkerung in Deutschland siehe beispielsweise den 10. Bericht der Beauftragten der Bundesregierung für Migration, Flüchtlinge und Integration über die Lage der Ausländerinnen und Ausländer in Deutschland (2014).

5 Für eine ausführliche Zusammenstellung entsprechender Studien siehe beispielsweise Hoffmann (2008), Kapitel 4. 


\section{Danksagung}

Wir bedanken uns bei Olga Pötzsch, Michael Mühlichen und bei zwei anonymen Gutachtern für wichtige Hinweise zu früheren Versionen dieser Arbeit.

\section{Literatur}

Balducci, Gaetano 1917: Costruzione e critica delle tavole di mortalità. In: Giornale degli Economisti e Rivista di Statistica, Serie terza, 55: 455-484.

Becker, Karl 1874: Zur Berechnung von Sterbetafeln an die Bevölkerungsstatistik zu stellende Anforderungen. Berlin.

Die Beauftragte der Bundesregierung für Migration, Flüchtlinge und Integration (Hrsg.) 2014: 10. Bericht der Beauftragten der Bundesregierung für Migration, Flüchtlinge und Integration über die Lage der Ausländerinnen und Ausländer in Deutschland [http:// www.bundesregierung.de/Content/Infomaterial/BPA/IB/10_Auslaenderbericht_2015. pdf, 08.06.2016].

Eilers, Paul H.C.; Marx, Brian D. 1996: Flexible Smoothing with B-splines and Penalties. In: Statistical Science 11,2: 89-121.

Eisenmenger, Matthias 2003: Die Erweiterung der abgekürzten Sterbetafel in den Altersstufen von 90 bis 100 Jahre. In: Sonderausgabe Wirtschaft und Statistik, ISI-Weltkongress: 90-92.

Eurostat 2015: Online-Datenbank - Tabelle Todesursachen - absolute Zahl im Berichtsland und für Einwohner (hlth_cd_aro) [http://appsso.eurostat.ec.europa.eu/nui/show. do?dataset=hlth_cd_aro, 08.0̄6.2016].

Fleischer, Henning 1989: Entwicklung der Ausländerzahl seit 1987. In: Wirtschaft und Statistik 9: 594-599.

Hoffmann, Rassmus 2008: Socioeconomic Differences in Old Age Mortality. In: Land, Kenneth $C$. (Hrsg.): The Springer series on demographic methods and population analysis 25. Springer, Dordrecht [doi: 10.1007/978-1-4020-8692-2].

Kaus, Wolfhard; Mundil-Schwarz, Rabea 2015: Die Ermittlung der Einwohnerzahlen und der demografischen Strukturen nach dem Zensus 2011. In: Wirtschaft und Statistik 4: 18-38.

Kennedy, Steven; Kidd, Michael P. 2015: The Healthy Immigrant Effect: Patterns and Evidence from Four Countries. In: Journal of International Migration and Integration 16,2: 317-332 [doi: 10.1007/s12134-014-0340-x].

Kibele, Eva; Scholz, Rembrandt; Shkolnikov, Vladimir M. 2008: Low migrant mortality in Germany for men aged 65 and older: fact or artifact? In: European Journal of Epidemiology 23: 289-393 [doi: 10.1007/s10654-008-9247-1].

Koh/s, Martin 2008a: Einfluss von Auswahlprozessen auf die Sterblichkeit verschiedener Zuwanderergruppen in Deutschland. In: DRV-Schriften 55: 153-175.

Koh/s, Martin 2008b: Leben Migranten wirklich länger? Eine empirische Analyse der Mortalität von Migranten in Deutschland. Bundesamt für Migration und Flüchtlinge, Working Paper 16.

Koh/s, Martin 2008c: Healthy-Migrant-Effect, Erfassungsfehler und andere Schwierigkeiten bei der Analyse der Mortalität von Migranten - Eine Bestandsaufnahme. Bundesamt für Migration und Flüchtlinge, Working Paper 15. 
Koh/s, Martin 2011: Morbidität und Mortalität von Migranten in Deutschland. Bundesamt für Migration und Flüchtlinge, Forschungsbericht 9.

Koh/s, Martin 2012: Demographie von Migranten in Deutschland. In: Razum, Oliver (Hrsg.): Challenges of public health 63. Frankfurt a.M.: Peter Lang.

Kohls, Martin 2015: Sterberisiken von Migranten - Analysen zum Healthy-Migrant-Effekt nach dem Zensus 2011. In: Bundesgesundheitsblatt 58/6: 519-526 [doi: 10.1007/ s00103-015-2140-7].

Luy, Marc 2007: Estimating the Migrant Survival Advantage from Orphanhood of Second Generation Migrants. Rostocker Zentrum zur Erforschung des demografischen Wandels, Rostocker Zentrum Diskussionspapier 17.

Mammey, Ulrich; Schwarz, Karl 2002: The demographic characteristics of the immigrant population in Germany. In: Haug, Werner; Compton, Paul; Courbage, Youssef (Hrsg.): The demographic characteristics of immigrant populations. Population Studies 38. Strassburg: Council of Europe: 193-244.

Moullan, Yasser; Jusot, Florence 2014: Why is the 'healthy immigrant effect' different between European countries? In: European Journal of Public Health 24,1: 80-86 [doi: 10.1093/eurpub/cku112].

Nielsen, Signe Smith; Krasnik, Allan 2010: Poorer self-perceived health among migrants and ethnic minorities versus the majority population in Europe: a systematic review. In: International Journal of Public Health 55,5: 357-371 [doi: 10.1007/s00038-010-0145-4].

Opfermann, Heike; Grobecker, Claire; Krack-Roberg, Elle 2006: Auswirkung der Bereinigung des Ausländerzentralregisters auf die amtliche Ausländerstatistik. In: Wirtschaft und Statistik 5: 480-494.

Perks, Wilfred 1932: On some experiments the graduation of mortality statistics. In: Journal of the Institute of Actuaries 63: 12-40.

Razum, Oliver 2009: Migration, Mortalität und der Healthy-migrant-Effekt. In: Richter, Matthias; Hurrelmann, Klaus (Hrsg.): Gesundheitliche Ungleichheit - Grundlagen, Probleme, Perspektiven. 2. aktualisierte Auflage. Wiesbaden: VS-Verlag: 268-282.

Razum, Oliver et al. 1998: Low overall mortality of Turkish residents in Germany persists and extends into a second generation: merely a healthy migrant effect? In: Tropical Medicine and International Health 3,4: 297-303 [doi: 10.1046/j.1365-3156.1998.00233.x].

Robert Koch Institut; Statistisches Bundesamt 2008: Schwerpunktbericht der Gesundheitsberichterstattung des Bundes: Migration und Gesundheit. Robert Koch Institut, Berlin [http://edoc.rki.de/documents/rki_fv/ren4T3cctjHcA/PDF/253bKE5YVJxo_28. pdf, 08.06.2016].

Salzmann, Thomas; Koh/s, Martin 2006: Mortalitätsanalyse mit den Daten der Deutschen Rentenversicherung - Methodische Überlegungen zum Stichprobencharakter. In: DRV-Schriften 55: 185-199.

Statistisches Bundesamt 2012: Periodensterbetafeln für Deutschland 2009/2011 [https://www.destatis.de/DE/Publikationen/Thematisch/Bevoelkerung/ Bevoelkerungsbewegung/PeriodensterbetafelnBundeslaender5126204117004.pdf, 08.06.2016].

Statistisches Bundesamt 2013: Bevölkerungsfortschreibung auf Grundlage der Volkszählung 1987 (Westen) bzw. 1990 (Osten) [https://www.destatis.de/DE/Publikationen/ Thematisch/Bevoelkerung/Bevoelkerungsstand/Bevoelkerungsfortschreibung.html, 08.06.2016]. 
Statistisches Bundesamt 2015a: Allgemeine Sterbetafel 2010/12 - Methodische Erläuterungen und Ergebnisse [https://www.destatis.de/DE/Publikationen/Thematisch/Bevoelkerung/Bevoelkerungsbewegung/SterbetafelnAllgemeinErlaeuterung5126205129004.pdf, 08.06.2016].

Statistisches Bundesamt 2015b: Einbürgerungen - Fachserie 1 Reihe 2.1 - 2014 [https:// www.destatis.de/DE/Publikationen/Thematisch/Bevoelkerung/MigrationIntegration/ Einbuergerungen.html, 08.06.2016].

Statistisches Bundesamt 2015c: Ausgangsdaten der Bevölkerungsfortschreibung aus dem Zensus [https://www.destatis.de/DE/Publikationen/Thematisch/Bevoelkerung/ Bevoelkerungsstand/DatenBevoelkerungsfortschreibungZensus.html, 08.06.2016].

Thatcher, A. Roger; Kannisto, Väinö; Vaupel, James W. 1998: The force of mortality at ages 80 to 120, Monographs on Population Aging, 5. Odense: Odense University Press.

Weber, Ingbert et al. 1990: Zur gesundheitlichen Lage der ausländischen Bevölkerung in der Bundesrepublik Deutschland: Erste Erkenntnisse. In: Projektgruppe Prioritäre Gesundheitsziele beim Zentralinstitut für die kassenärztliche Versorgung. Dringliche Gesundheitsprobleme in der Bundesrepublik Deutschland - Zahlen - Fakten - Perspektiven. Baden-Baden: Nomos Verlagsgesellschaft: 577-601.

Wittstein, Theodor 1862: Die Mortalität in Gesellschaften mit successiv eintretenden und ausscheidenden Mitgliedern. In: Archiv der Mathematik und Physik 39: 67-92.

Zeuner, Gustav 1869: Abhandlungen zur mathematischen Statistik. Leipzig.

zur Nieden, Felix; Rau, Roland; Luy, Marc 2016: Allgemeine Sterbetafel 2010/12 - Neue Ansätze zur Glättung und Extrapolation der Sterbewahrscheinlichkeiten. In: Wirtschaft und Statistik 1: 63-73.

Eine Übersetzung dieses begutachteten und von den Autoren autorisierten deutschen Originaltextes durch das Bundesinstitut für Bevölkerungsforschung ist unter dem Titel "Life Expectancy in Germany Based on the 2011 Census: Was the Healthy Migrant Effect Merely an Artefact?", DOI 10.12765/CPoS-2016-05en bzw. URN urn:nbn:de:bib-cpos-2016-05en0, auf http://www.comparativepopulationstudies. de verfügbar.

Eingegangen am: 14.10.2015

Angenommen am: 21.03.2016

Felix zur Nieden $(\bowtie)$, Bettina Sommer. Statistisches Bundesamt (Destatis). Wiesbaden, Deutschland. E-Mail: felix.zurnieden@destatis.de, bettina.sommer@destatis.de URL: https://www.destatis.de/DE/Startseite.html 


\section{Comparative Population Studies}

wWW.comparativepopulationstudies.de

ISSN: 1869-8980 (Print) - 1869-8999 (Internet)

\section{Published by}

Prof. Dr. Norbert F. Schneider

Federal Institute for Population Research D-65180 Wiesbaden / Germany

\section{(cc) BY-SA}

2017

\section{Managing Editor}

Frank Swiaczny

\section{Assistant Managing Editor}

Katrin Schiefer

\section{Copy Editor}

(Selected Articles in German)

Dr. Evelyn Grünheid

\section{Layout}

Beatriz Feiler-Fuchs

E-mail: cpos@bib.bund.de

\section{Scientific Advisory Board}

Paul Gans (Mannheim)

Karsten Hank (Cologne)

Johannes Huinink (Bremen)

Michaela Kreyenfeld (Rostock)

Marc Luy (Vienna)

Notburga Ott (Bochum)

Peter Preisendörfer (Mainz)

Nikola Sander (Groningen)

Zsolt Spéder (Budapest)

\section{Board of Reviewers}

Martin Abraham (Erlangen)

Laura Bernardi (Lausanne)

Hansjörg Bucher (Bonn)

Claudia Diehl (Konstanz)

Andreas Diekmann (Zurich)

Gabriele Doblhammer-Reiter (Rostock)

Jürgen Dorbritz (Wiesbaden)

Anette Eva Fasang (Berlin)

E.-Jürgen Flöthmann (Bielefeld)

Alexia Fürnkranz-Prskawetz (Vienna)

Beat Fux (Salzburg)

Joshua Goldstein (Berkeley)

Sonja Haug (Regensburg)

Hill Kulu (Liverpool)

Aart C. Liefbroer (The Hague)

Kurt Lüscher (Konstanz)

Emma Lundholm (Umeå)

Nadja Milewski (Rostock)

Dimiter Philipov (Vienna)

Roland Rau (Rostock)

Tomáš Sobotka (Vienna)

Jeroen Spijker (Barcelona)

Olivier Thévenon (Paris)

Helga de Valk (Brussels)

Heike Trappe (Rostock)

Michael Wagner (Cologne) 